\title{
Two-Scale Convergence of First-Order Operators
}

\author{
Augusto Visintin
}

\begin{abstract}
Nguetseng's notion of two-scale convergence and some of its main properties are first shortly reviewed. The (weak) two-scale limit of the gradient of bounded sequences of $W^{1, p}\left(\mathbb{R}^{N}\right)$ is then studied: if $u_{\varepsilon} \rightarrow u$ weakly in $W^{1, p}\left(\mathbb{R}^{N}\right)$, a sequence $\left\{u_{1 \varepsilon}\right\}$ is constructed such that $u_{1 \varepsilon}(x) \rightarrow u_{1}(x, y)$ and $\nabla u_{\varepsilon}(x) \rightarrow \nabla u(x)+\nabla_{y} u_{1}(x, y)$ weakly two-scale. Analogous constructions are introduced for the weak two-scale limit of derivatives in the spaces $W^{1, p}\left(\mathbb{R}^{N}\right)^{N}, L_{\text {rot }}^{2}\left(\mathbb{R}^{3}\right)^{3}, L_{\text {div }}^{2}\left(\mathbb{R}^{N}\right)^{N}, L_{\text {div }}^{2}\left(\mathbb{R}^{N}\right)^{N^{2}}$. The application to the two-scale limit of some classical equations of electromagnetism and continuum mechanics is outlined. These results are then applied to the homogenization of quasilinear elliptic equations like $\nabla \times\left[A\left(u_{\varepsilon}(x), x, \frac{x}{\varepsilon}\right) \cdot \nabla \times u_{\varepsilon}\right]=f$.
\end{abstract}

Keywords. Two-scale convergence, homogenization, elliptic equations, electromagnetism, continuum mechanics

Mathematics Subject Classification (2000). Primary 35B27, secondary 74Q, $78 \mathrm{M} 40$

\section{Introduction}

The following notion was introduced by Nguetseng [27], and then developed by Allaire [1] and others: a bounded sequence $\left\{u_{\varepsilon}\right\}$ of $L^{2}\left(\mathbb{R}^{N}\right)$ is said (weakly) two-scale convergent to $u \in L^{2}\left(\mathbb{R}^{N} \times Y\right)$ (with $Y:=\left[0,1\left[^{N}\right)\right.$ if and only if

$$
\lim _{\varepsilon \rightarrow 0} \int_{\mathbb{R}^{N}} u_{\varepsilon}(x) \psi\left(x, \frac{x}{\varepsilon}\right) d x=\iint_{\mathbb{R}^{N} \times Y} u(x, y) \psi(x, y) d x d y,
$$

for any smooth function $\psi: \mathbb{R}^{N} \times \mathbb{R}^{N} \rightarrow \mathbb{R}$ that is $\mathrm{Y}$-periodic w.r.t. the second argument and such that $\psi \in L^{2}\left(\mathbb{R}^{N} \times Y\right)$. (This is easily extended to $L^{p}$ for any $p \in\left[1,+\infty[\right.$.$) It should be noticed that the converging functions u_{\varepsilon}$ only depend on $x$, whereas the limit function $u$ may also depend on the variable $y$; $x$ and $y$ may respectively be regarded as coarse-scale and fine-scale variables. Two-scale convergence can thus account for occurrence of a fine-scale periodic

A. Visintin: Department of Mathematics, University of Trento, Italy; Visintin@science.unitn.it 
structure, and indeed has been applied to a number of homogenization problems, see e.g. $[1,4,8,12,15,18,28,42]$. For periodic homogenization, this method is indeed alternative to the classical energy method (or method of oscillating test functions) due to Tartar, see e.g. [2, 6, 14, 19, 23, 26], [29]-[33].

In this paper we deal with the two-scale convergence of some first-order linear differential operators (with constant coefficients). First we briefly review some basic definitions and results, along the lines of $[13,34,35]$ and of other works. We then revisit a known result: if $\left\{u_{\varepsilon}\right\}$ is a sequence of $W^{1, p}\left(\mathbb{R}^{N}\right)$ $(1<p<+\infty)$ and $u_{\varepsilon} \rightarrow u$ weakly in this space, then there exists a (possibly nonunique) function $u_{1} \in L^{p}\left(\mathbb{R}^{N} ; W^{1, p}(\mathcal{Y})\right)$ (Y) being the $N$-dimensional unit torus) such that, up to a subsequence,

$$
\nabla u_{\varepsilon} \rightarrow \nabla u+\nabla_{y} u_{1} \quad \text { weakly two-scale in } L^{p}\left(\mathbb{R}^{N} \times \mathcal{Y}\right)^{N} .
$$

This result was already proved in [27] and [1]. Here we investigate how $u_{1}$ may be related to the sequence $\left\{u_{\varepsilon}\right\}$ : in Theorem 2.2 we construct a sequence $\left\{u_{1 \varepsilon}\right\}$ such that, up to a subsequence,

$$
\begin{aligned}
u_{1 \varepsilon} & \rightarrow u_{1} & & \text { weakly two-scale in } L^{p}\left(\mathbb{R}^{N} \times \mathcal{Y}\right) \\
\varepsilon \nabla u_{1 \varepsilon} & \rightarrow \nabla_{y} u_{1} & & \text { weakly two-scale in } L^{p}\left(\mathbb{R}^{N} \times \mathcal{Y}\right)^{N} .
\end{aligned}
$$

For any $\varepsilon$, the function $u_{1 \varepsilon}$ solves an elliptic periodic problem on each cell $\varepsilon(m+Y)$, with $m \in \mathbb{Z}^{N}$. This result may be compared with Theorem 1 of [13], which outlines a different approximation of the function $u_{1}$. In addition we show that conversely for any pair $\left(u, u_{1}\right)$ as above there exists a sequence $\left\{u_{\varepsilon}\right\}$ for which (2) is fulfilled, here with strong two-scale convergence. The stated regularity of $u$ and $u_{1}$ may thus be regarded as optimal.

There are analogies between our construction of $u_{1 \varepsilon}$ and the so-called cell problem, that is at the basis of the homogenization of the elliptic equation $-\sum_{i, j=1}^{N} a_{i j}\left(\frac{x}{\varepsilon}\right) D_{i} D_{j} u_{\varepsilon}=f$ in $\mathbb{R}^{N}\left(D_{j}:=\frac{\partial}{\partial x_{j}}\right)$, the matrix function $\left\{a_{i j}\right\}$ being $Y$-periodic and positive-definite. Elliptic equations like this have extensively been studied in a large literature, via asymptotic expansions and Tartar's energy method; see e.g. the abovementioned monographs. There is however a major difference between the two settings: the classical cell problem involves the elliptic operator, whereas here it only depends on the sequence $\left\{u_{\varepsilon}\right\}$, and is not related to any specific problem.

The main aim of this paper is to derive analogous results for other firstorder linear differential operators (with constant coefficients), by extending the construction of Section 2. Arguments relative to different operators exhibit similarities but also several differences; we then develop them in detail. In Section 3 for any fixed $p \in] 1,+\infty[$ we deal with sequences of the space

$$
\left\{v \in L^{p}\left(\mathbb{R}^{N}\right)^{N}:\left(\nabla^{s} u\right)_{i j}:=\frac{1}{2}\left(D_{j} v_{i}+D_{i} v_{j}\right) \in L^{p}\left(\mathbb{R}^{N}\right), i, j=1, \ldots, N\right\},
$$


that we equip with the graph norm. By the classical Korn inequality this space coincides with $W^{1, p}\left(\mathbb{R}^{N}\right)^{N}$. Given a weakly convergent sequence in this space, we construct a function $u_{1} \in L^{p}\left(\mathbb{R}^{N} ; W^{1, p}(\mathcal{Y})^{N}\right)$, a two-scale approximating sequence $\left\{u_{1 \varepsilon}\right\}$, and derive statements like (2) and (3), with the symmetrized gradient, $\nabla^{s}$, in place of the gradient, $\nabla$. A converse statement for strong two-scale convergence holds for this as well as for the other extensions that follow. This theorem looks prone to applications to continuum mechanics, for the linearized strain is the symmetrized gradient of the displacement.

In the remainder of this paper we assume that $p=2$. In view of applications to electromagnetism, in Sections 4 and 5 we deal with sequences of $L_{\text {rot }}^{2}\left(\mathbb{R}^{3}\right)^{3}$ and of $L_{\text {div }}^{2}\left(\mathbb{R}^{N}\right)^{N}$ (respectively the domain of the operators curl and divergence in $L^{2}$ ), and derive formulas analogous to (2) and (3). Dealing with $L_{\text {div }}^{2}$ we assume that $N$ is any positive integer. On the other hand we study convergence in $L_{\text {rot }}^{2}$ just for $N=3$, which is the case of main applicative interest, for the extension to $N>3$ would be slightly cumbersome. In Section 6 we prove similar results for sequences of the space

$$
L_{\text {div }}^{2}\left(\mathbb{R}^{3}\right)^{9}:=\left\{v \in L^{2}\left(\mathbb{R}^{3}\right)_{s}^{9}: \sum_{j=1}^{3} D_{j} v_{i j} \in L^{2}\left(\mathbb{R}^{3}\right)(i=1,2,3)\right\},
$$

that we equip with the graph norm (the index $s$ restricts the space to symmetric tensors). The latter setting is of potential interest for applications to continuum mechanics, for the stress tensor is a typical element of that space, cf. [37], [38].

Despite of several analogies, Sections 4, 5, 6 differ from Sections 2, 3 in the following respect. Weak convergence in $W^{1, p}\left(\mathbb{R}^{N}\right)$ entails strong convergence in $L_{\text {loc }}^{p}\left(\mathbb{R}^{N}\right)$; hence the weak two-scale limit does not depend on $y$ and coincides with the strong one-scale limit. On the other hand, weak convergence in $L_{\text {rot }}^{2}\left(\mathbb{R}^{3}\right)^{3}$ does not entail strong convergence in $L_{\text {loc }}^{2}\left(\mathbb{R}^{3}\right)^{3}$; hence the weak twoscale limit $u$ may also depend on the fine-scale variable $y$, and thus differ from the weak one-scale limit $\bar{u}:=\int_{\mathcal{Y}} u(\cdot, y) d y$. This raises the question whether, assuming that $u_{\varepsilon} \rightarrow u$ weakly two-scale in $L^{2}\left(\mathbb{R}^{3} \times \mathcal{Y}\right)^{3}$, the weak two-scale limit of $\nabla \times u_{\varepsilon}(\nabla \times:=$ curl $)$ should read

$$
\nabla \times \bar{u}(x)+\nabla_{y} \times u_{1}(x, y) \quad \text { or } \quad \nabla_{x} \times u(x, y)+\nabla_{y} \times \tilde{u}_{1}(x, y)
$$

We derive the former expression; this looks fairly natural, for it confines any dependence on the fine-scale variable $y$ to the correcting term $\nabla_{y} \times u_{1}$. Anyway this is compatible with the second expression, which indeed has recently been studied by Wellander and Kristensson [41] and used in [7] (analogous results might be proved for the other first-order differential operators we deal with in this paper). A similar remark applies to the results of Sections 5 and 6, dealing with the divergence operator; also in this case the formula that we prove includes the derivatives of $\bar{u}$, and the two-scale limit reads $\nabla \cdot \bar{u}+\nabla_{y} \cdot u_{1}(\nabla \cdot:=\operatorname{div})$. It may also be noticed that in all of these cases (for $p=2$ ) we represent the weak 
two-scale limit of the differential operator as the sum of two orthogonal terms of $L^{2}\left(\mathbb{R}^{N} \times \mathcal{Y}\right)^{M}$ ( $M$ depending on the specific setting).

In Section 7 we illustrate how these results may be applied to the study of the two-scale limit of the classical equations of Ampère and Gauss of electromagnetism, and of the balance of momentum of continuum mechanics.

The above developments may be used in the homogenization of several second-order elliptic equations, for the corresponding a priori estimates are typically expressed in terms of first-order operators like gradient, curl or divergence. In Section 8 we deal with the asymptotic behaviour of the system

$$
\nabla \times\left[A\left(u_{\varepsilon}(x), x, \frac{x}{\varepsilon}\right) \cdot\left(\nabla \times u_{\varepsilon}+f\right)\right]=0, \quad \nabla \cdot u_{\varepsilon}=0 \quad \text { in } \Omega,
$$

coupled with homogeneous Dirichlet conditions. This may represent equilibrium in a heterogeneous and anisotropic electric conductor with a magnetic-fielddependent resistance $A$. Here we prove convergence to a two-scale solution. Analogous conclusions might be reached for instance for the equation $-\nabla$. $\left[A\left(u_{\varepsilon}(x), x, \frac{x}{\varepsilon}\right) \cdot \nabla u_{\varepsilon}\right]=f$. The homogenization of quasilinear elliptic equations in divergence form has been studied in a large number of papers, see e.g. $[9,16]$ and references therein; two-scale convergence has been applied e.g. in $[1,10$, $13,27]$. But apparently so far less attention has been paid to equations in curl form, and to corresponding variational problems.

This work is part of a research on two-scale convergence. Some of these results have been announced in [34]; see also [35]. This author intends to apply them to the homogenization of nonlinear partial differential equations issued from electromagnetism, phase transitions and continuum mechanics [36]-[39]. Further research will deal with the two-scale homogenization of elliptic operators.

\section{Two-scale convergence of the gradient of a scalar field}

Two-scale decomposition. In this section we briefly review some properties of two-scale convergence, along the lines of Nguetseng [27] and Allaire [1]. Here we use the formulation of $[34,35]$, that is based on a technique that has already been investigated in several papers, cf. e.g. [3, 4, 8, 12, 13, 21, 22].

We set $Y=\left[0,1\left[^{N}\right.\right.$, denote by $\mathcal{Y}$ the same set equipped with the topological and differential structure of the $N$-dimensional unit torus. (The case of a cell of the form $[0, a[\times[0, b[\times[0, c[$ might be dealt similarly. $)$ We identify any function on $\mathcal{Y}$ with its $Y$-periodic extension to $\mathbb{R}^{N}$. For any $\varepsilon>0$ we set

$$
\begin{aligned}
& \hat{n}(x):=\max \{n \in \mathbb{Z}: n \leq x\}, \quad \hat{r}(x):=x-\hat{n}(x)(\in[0,1[) \quad \forall x \in \mathbb{R} \\
& \mathcal{N}(x):=\left(\hat{n}\left(x_{1}\right), \ldots, \hat{n}\left(x_{N}\right)\right) \in \mathbb{Z}^{N}, \quad \mathcal{R}(x):=x-\mathcal{N}(x) \in \mathcal{Y} \quad \forall x \in \mathbb{R}^{N} ;
\end{aligned}
$$


this yields the two-scale decomposition (also named periodic unfolding in [13]): $x=\varepsilon\left[\mathcal{N}\left(\frac{x}{\varepsilon}\right)+\mathcal{R}\left(\frac{x}{\varepsilon}\right)\right]$ for any $x \in \mathbb{R}^{N} ; \varepsilon \mathcal{N}\left(\frac{x}{\varepsilon}\right)$ and $\mathcal{R}\left(\frac{x}{\varepsilon}\right)$ may respectively be regarded as coarse-scale and fine-scale variables w.r.t. the scale $\varepsilon$. We also set

$$
S_{\varepsilon}(x, y):=\varepsilon \mathcal{N}\left(\frac{x}{\varepsilon}\right)+\varepsilon y \quad \forall(x, y) \in \mathbb{R}^{N} \times \mathcal{Y}, \forall \varepsilon>0 .
$$

The next statement can easily be proved via a variable transformation. Let us first denote by $\mathcal{L}\left(\mathbb{R}^{N}\right)\left(\mathcal{B}\left(\mathbb{R}^{N}\right)\right.$, resp.) the $\sigma$-algebra of Lebesgue- (Borel-, resp.) measurable subsets of $\mathbb{R}^{N}$, and define $\mathcal{L}(\mathcal{Y})$ and $\mathcal{B}(\mathcal{Y})$ similarly.

Lemma $1.1([35])$. Let $f: \mathbb{R}^{N} \times \mathcal{Y} \rightarrow \mathbb{R}$ be such that

$f$ is measurable either w.r.t. the $\sigma$-algebra generated by $\mathcal{B}\left(\mathbb{R}^{N}\right) \times \mathcal{L}(\mathcal{Y})$, or w.r.t. that generated by $\mathcal{L}\left(\mathbb{R}^{N}\right) \times \mathcal{B}(\mathcal{Y})$,

either $\|f\|_{L^{\infty}\left(\mathbb{R}^{N}\right)} \in L^{1}(\mathcal{Y})$ and has compact support, or $\|f\|_{L^{\infty}(\mathcal{Y})} \in L^{1}\left(\mathbb{R}^{N}\right)$.

Let us extend $f(x, \cdot)$ by $Y$-periodicity to $\mathbb{R}^{N}$ for a.a. $x \in \mathbb{R}^{N}$. Then, for any $\varepsilon>0$, the functions $\mathbb{R}^{N} \rightarrow \mathbb{R}: x \mapsto f\left(x, \frac{x}{\varepsilon}\right)$ and $\mathbb{R}^{N} \times \mathcal{Y} \rightarrow \mathbb{R}:(x, y) \mapsto f\left(S_{\varepsilon}(x, y), y\right)$ are integrable, and

$$
\begin{aligned}
\int_{\mathbb{R}^{N}} f\left(x, \frac{x}{\varepsilon}\right) d x & =\varepsilon^{N} \sum_{m \in \mathbb{Z}^{N}} \int_{Y} f(\varepsilon[m+y], y) d y \\
& =\iint_{\mathbb{R}^{N} \times Y} f\left(S_{\varepsilon}(x, y), y\right) d x d y \quad \forall \varepsilon>0 .
\end{aligned}
$$

For any $p \in[1,+\infty]$ and $\varepsilon>0$, the operator $g \mapsto g \circ S_{\varepsilon}$ is then a (nonsurjective) linear isometry $L^{p}\left(\mathbb{R}^{N}\right) \rightarrow L^{p}\left(\mathbb{R}^{N} \times \mathcal{Y}\right)$.

Two-Scale Convergence in $L^{p}\left(\mathbb{R}^{N} \times \mathcal{Y}\right)$. In this paper, by $\varepsilon$ we shall denote the generic element of an arbitrary but prescribed, vanishing sequence of positive real numbers; e.g. $\varepsilon=\left\{1, \frac{1}{2}, \frac{1}{3}, \ldots, \frac{1}{n}, \ldots\right\}$. Let $p \in[1,+\infty]$; for any sequence $\left\{u_{\varepsilon}\right\}$ of $L^{p}\left(\mathbb{R}^{N}\right)$ and any $u \in L^{p}\left(\mathbb{R}^{N^{n}} \times \mathcal{Y}\right)$, we say that $u_{\varepsilon}$ strongly twoscale converges to $u$ in $L^{p}\left(\mathbb{R}^{N} \times \mathcal{Y}\right)$, whenever $u_{\varepsilon} \circ S_{\varepsilon} \rightarrow u$ strongly in the latter space. We similarly define weak (weak star for $p=\infty$ ) two-scale convergence. We denote these convergences by $u_{\varepsilon} \underset{2}{\rightarrow} u, u_{\varepsilon} \underset{2}{\overrightarrow{2}} u, u_{\varepsilon} \frac{*}{2} u$, resp., and reserve the symbols $\rightarrow, \rightarrow$, $*$ for the ordinary strong, weak, weak star convergence (that we refer to as one-scale convergence). Thus for any $p \in[1,+\infty]$

$$
\begin{aligned}
& u_{\varepsilon} \underset{2}{\rightarrow} u \text { in } L^{p}\left(\mathbb{R}^{N} \times \mathcal{Y}\right) \Leftrightarrow u_{\varepsilon} \circ S_{\varepsilon} \rightarrow u \text { in } L^{p}\left(\mathbb{R}^{N} \times \mathcal{Y}\right) \\
& u_{\varepsilon} \overrightarrow{2} u \text { in } L^{p}\left(\mathbb{R}^{N} \times \mathcal{Y}\right) \Leftrightarrow u_{\varepsilon} \circ S_{\varepsilon} \rightarrow u \text { in } L^{p}\left(\mathbb{R}^{N} \times \mathcal{Y}\right) \\
& u_{\varepsilon} \frac{*}{2} u \text { in } L^{\infty}\left(\mathbb{R}^{N} \times \mathcal{Y}\right) \Leftrightarrow u_{\varepsilon} \circ S_{\varepsilon} \stackrel{*}{\rightarrow} u \text { in } L^{\infty}\left(\mathbb{R}^{N} \times \mathcal{Y}\right) \text {. }
\end{aligned}
$$


For any domain $\Omega \subset \mathbb{R}^{N}$, we then define two-scale convergence in $L^{p}(\Omega \times \mathcal{Y})$ by extending functions to $\mathbb{R}^{N} \backslash \Omega$ with vanishing value. These definitions slightly extend the original ones of Nguetseng [27] and Allaire [1]; see [35]. The next result is a simple consequence of the above construction.

Proposition $1.2([1,27])$. Let $p \in] 1,+\infty]$. For any bounded sequence $\left\{u_{\varepsilon}\right\}$ of $L^{p}\left(\mathbb{R}^{N}\right)$ there exists $u \in L^{p}\left(\mathbb{R}^{N} \times \mathcal{Y}\right)$ such that, up to a subsequence, $u_{\varepsilon} \underset{2}{\vec{u}}$ in $L^{p}\left(\mathbb{R}^{N}\right)\left(u_{\varepsilon} \frac{*}{2} u\right.$ if $\left.p=\infty\right)$.

In view of the next statement let us fix a radial function $\rho$ such that $\rho \in$ $C^{1}\left(\mathbb{R}^{N}\right), \rho \geq 0, \rho(x)=0$ if $|x| \geq 1, \int_{\mathbb{R}^{N}} \rho(x) d x=1$, and set

$$
\rho_{\varepsilon}(x):=\varepsilon^{-\frac{N}{2}} \rho\left(\varepsilon^{-\frac{1}{2}} x\right) \quad \forall x \in \mathbb{R}^{N} .
$$

Hence $\nabla \rho_{\varepsilon}(x)=\varepsilon^{-\frac{N+1}{2}}(\nabla \rho)\left(\varepsilon^{-\frac{1}{2}} x\right)$ and $\rho_{\varepsilon} * \delta_{0}$ (the Dirac measure) in $C^{0}\left(\mathbb{R}^{N}\right)^{\prime}$. For any function $u=u(x, y)$ henceforth we set

$$
\bar{u}:=\int_{\mathcal{Y}} u(\cdot, y) d y, \quad \tilde{u}:=u-\bar{u} \quad \text { a.e. in } \mathbb{R}^{N} \times \mathcal{Y} .
$$

Proposition 1.3. Let $p \in\left[1,+\infty\left[\right.\right.$ and $\left\{u_{\varepsilon}\right\}$ be a sequence in $L^{p}\left(\mathbb{R}^{N}\right)$ such that $u_{\varepsilon} \underset{2}{\rightarrow} u$ in $L^{p}\left(\mathbb{R}^{N} \times \mathcal{Y}\right)$. Then

$$
u_{\varepsilon}-\int_{\mathbb{R}^{N}} \tilde{u}\left(\xi, \frac{x}{\varepsilon}\right) \rho_{\varepsilon}(x-\xi) d \xi \rightarrow \bar{u} \quad \text { in } L^{p}\left(\mathbb{R}^{N}\right) .
$$

Proof. By Proposition 2.13 of $[35] \int_{\mathbb{R}^{N}} \tilde{u}\left(\xi, \frac{x}{\varepsilon}\right) \rho_{\varepsilon}(x-\xi) d \xi \underset{2}{\longrightarrow} \tilde{u}$ in $L^{p}\left(\mathbb{R}^{N} \times \mathcal{Y}\right)$. By the hypotheses the same then applies to the sequence of (1.8). It then suffices to notice that, for any sequence $\left\{w_{\varepsilon}\right\}$ in $L^{p}\left(\mathbb{R}^{N}\right)$ and any $w \in L^{p}\left(\mathbb{R}^{N}\right), w_{\varepsilon} \rightarrow w$ in $L^{p}\left(\mathbb{R}^{N}\right)$ if and only if $w_{\varepsilon} \underset{2}{\longrightarrow} w$ in $L^{p}\left(\mathbb{R}^{N} \times \mathcal{Y}\right)$.

Further Notation. By appending the index $*$ to a space of functions over $\mathcal{Y}$, we shall denote the subspace of functions having vanishing mean. For any function of $(x, y) \in \mathbb{R}^{N} \times \mathcal{Y}$, we shall denote by $\nabla_{x}\left(\nabla_{y}\right.$, resp. $)$ the gradient w.r.t. to the first (second, resp.) vector argument. Finally expressions like $\nabla u v$ should be interpreted as $(\nabla u) v$ (this will make formulas look a bit lighter).

\section{Two-scale convergence of the gradient of a scalar field}

In this section we deal with the two-scale limit of the gradient of weakly convergent sequences of $W^{1, p}\left(\mathbb{R}^{N}\right)$, for any $\left.p \in\right] 1,+\infty[$ and $N \in \mathbb{N}$. Trivial examples show that $u_{\varepsilon} \rightarrow u$ in $W^{1, p}\left(\mathbb{R}^{N}\right)$ does not entail $\nabla u_{\varepsilon} \overrightarrow{2}_{2} \nabla_{x} u$ in $L^{p}\left(\mathbb{R}^{N} \times \mathcal{Y}\right)^{N}$ : in general the weak two-scale limit of the gradient need not coincide either with the weak one-scale limit of the gradient or with the gradient of the weak one-scale limit. 
In view of the next statement, for any Banach space $B$ let us denote by $\ell^{p}(B)$ the Banach space of $p$-summable functions $\mathbb{Z}^{N} \rightarrow B$.

Lemma 2.1 (Cell Projection). Let $p \in] 1,+\infty[$ and $\varepsilon>0$. For any $w \in$ $W^{1, p}\left(\mathbb{R}^{N}\right)$ there exists a unique $w^{*}=w^{*}(m, y) \in \ell^{p}\left(W_{*}^{1, p}(\mathcal{Y})\right)$ such that

$$
\begin{gathered}
\int_{\mathcal{Y}}\left[\nabla w^{*}(m, y)-(\nabla w)(\varepsilon(m+y))\right] \cdot \nabla \zeta d y=0 \\
\forall \zeta \in W^{1, p^{\prime}}(\mathcal{Y})\left(p^{\prime}:=\frac{p}{p-1}\right), \quad \forall m \in \mathbb{Z}^{N} .
\end{gathered}
$$

Moreover there exists a constant $C_{1}>0$ (independent of $w$ and $\varepsilon$ ) such that

$$
\begin{aligned}
& \varepsilon^{\frac{N}{p}}\left\|\nabla w^{*}\right\|_{\ell^{p}\left(L^{p}(\mathcal{Y})^{N}\right)}\left.=\left(\varepsilon^{N} \sum_{m \in \mathbb{Z}^{N}}\left\|\nabla w^{*}(m, \cdot)\right\|_{L^{p}(\mathcal{Y})^{N}}^{p}\right)^{\frac{1}{p}}\right) \\
& \leq\|\nabla w\|_{L^{p}\left(\mathbb{R}^{N}\right)^{N}} \\
& \varepsilon^{\frac{N}{p}}\left\|w^{*}\right\|_{\ell^{p}\left(L^{p}(\mathcal{Y})\right)} \leq C_{1}\|\nabla w\|_{L^{p}\left(\mathbb{R}^{N}\right)^{N} .}
\end{aligned}
$$

Notice that $(\nabla w)(\varepsilon(m+y)):=\left.\nabla w(x)\right|_{x=\varepsilon(m+y)}=\varepsilon^{-1} \nabla_{y}[w(\varepsilon(m+y))]$. Here $\nabla w^{*}(m, \cdot)\left(\nabla w\right.$, resp.) is the gradient in the sense of $\mathcal{D}^{\prime}(\mathcal{Y})^{N}\left(\right.$ of $\mathcal{D}^{\prime}\left(\mathbb{R}^{N}\right)^{N}$, resp.). Having identified $Y$ to $\mathcal{Y}, L^{p}(Y)^{N}=L^{p}(\mathcal{Y})^{N}$, but $W^{1, p}(Y) \neq W_{*}^{1, p}(\mathcal{Y})$.

Leaving aside translations and rescalings, the equation $\int_{\mathcal{Y}}(\nabla z-\nabla v) \cdot \nabla \zeta d y=$ 0 (for any $\zeta \in W^{1, p^{\prime}}(\mathcal{Y})$ ) defines a continuous projection (namely a continuous, linear and idempotent operator) $L: W^{1, p}(Y) \mapsto W_{*}^{1, p}(\mathcal{Y}): v \mapsto z$.

Proof. Let us fix any $m \in \mathbb{Z}^{N}$ and set $g:=(\nabla w)(\varepsilon(m+\cdot)) \in L^{p}(Y)^{N}$. For $p=2$ the equation (2.1) is equivalent to minimizing the functional

$$
H_{*}^{1}(\mathcal{Y}) \rightarrow \mathbb{R}: v \mapsto \int_{\mathcal{Y}}\left(\frac{1}{2}|\nabla v|^{2}-g \cdot \nabla v\right) d y
$$

existence and uniqueness of $w_{m}^{*}:=w^{*}(m, \cdot)$ are then straightforward. For $p \neq 2$ this setting does not fit the standard framework because of periodicity; we then provide a direct argument for existence and derive a uniform estimate.

If $g \in \mathcal{D}(\mathcal{Y})$, then there exists a unique $w_{m}^{*} \in H_{*}^{1}(\mathcal{Y})$ such that

$$
\int_{\mathcal{Y}}\left(\nabla w_{m}^{*}-g\right) \cdot \nabla v d y=0 \quad \forall v \in H^{1}(\mathcal{Y})
$$

actually $w_{m}^{*} \in \mathcal{D}_{*}(\mathcal{Y})$ by a known result of local regularity and by the compactness of $\mathcal{Y}$. Similarly there exists a unique $\psi \in \mathcal{D}_{*}(\mathcal{Y})$ such that

$$
\int_{\mathcal{Y}}\left(\nabla \psi-\left|\nabla w_{m}^{*}\right|^{p-2} \nabla w_{m}^{*}\right) \cdot \nabla v d y=0 \quad \forall v \in H^{1}(\mathcal{Y}) .
$$


By taking first $v=w_{m}^{*}$, then $v=\nabla w(\varepsilon(m+\cdot))$ in the latter formula, and then $v=\psi$ in (2.4), we get

$$
\begin{aligned}
\int_{\mathcal{Y}} \nabla \psi \cdot \nabla w_{m}^{*} d y & =\int_{\mathcal{Y}}\left|\nabla w_{m}^{*}\right|^{p} d y \\
\int_{\mathcal{Y}} \nabla \psi \cdot g d y & =\int_{\mathcal{Y}}\left|\nabla w_{m}^{*}\right|^{p-2} \nabla w_{m}^{*} \cdot g d y \\
\int_{\mathcal{Y}} \nabla w_{m}^{*} \cdot \nabla \psi d y & =\int_{\mathcal{Y}} g \cdot \nabla \psi d y .
\end{aligned}
$$

The three latter formulas yield

$$
\int_{\mathcal{Y}}\left|\nabla w_{m}^{*}\right|^{p} d y=\int_{\mathcal{Y}}\left|\nabla w_{m}^{*}\right|^{p-2} \nabla w_{m}^{*} \cdot g d y \leq\left\|\nabla w_{m}^{*}\right\|_{L^{p}(\mathcal{Y})^{N}}^{p-1}\|g\|_{L^{p}(\mathcal{Y})^{N}},
$$

whence

$$
\left\|\nabla w_{m}^{*}\right\|_{L^{p}(\mathcal{Y})^{N}} \leq\|g\|_{L^{p}(\mathcal{Y})^{N}}=\|(\nabla w)(\varepsilon(m+\cdot))\|_{L^{p}(\mathcal{Y})^{N}} \quad \forall m \in \mathbb{Z}^{N} .
$$

The restriction to $\mathcal{D}(\mathcal{Y})$ of the linear mapping $w(\varepsilon(m+\cdot)) \mapsto w_{m}^{*}$ is thus nonexpansive w.r.t. the $W_{*}^{1, p}$-seminorm. It can then be extended by density to a unique mapping $W^{1, p}(Y) \rightarrow W_{*}^{1, p}(\mathcal{Y})$. By (2.5) Lemma 1.1 yields

$$
\begin{aligned}
\varepsilon^{\frac{N}{p}}\left\|\nabla w^{*}\right\|_{\ell^{p}\left(L^{p}(\mathcal{Y})^{N}\right)} & =\left(\varepsilon^{N} \sum_{m \in \mathbb{Z}^{N}}\left\|\nabla w^{*}(m, \cdot)\right\|_{L^{p}(\mathcal{Y})^{N}}^{p}\right)^{\frac{1}{p}} \\
& \leq\left(\varepsilon^{N} \sum_{m \in \mathbb{Z}^{N}}\|(\nabla w)(\varepsilon(m+\cdot))\|_{L^{p}(\mathcal{Y})^{N}}^{p}\right)^{\frac{1}{p}} \\
& =\|\nabla w\|_{L^{p}\left(\mathbb{R}^{N}\right)^{N}}
\end{aligned}
$$

namely (2.2). By the classical Poincaré inequality, there exists a constant $C_{1}>0$ independent of $w$ and $\varepsilon$ such that $\int_{\mathcal{Y}}\left|w_{m}^{*}\right|^{p} d y \leq C_{1} \int_{\mathcal{Y}}\left|\nabla w_{m}^{*}\right|^{p} d y$. (2.5) then yields (2.3). The uniqueness of the solution of (2.1) follows from (2.3).

The first part of the next result may be compared with analogous theorems of $[1,27]$ for $p=2$; more recently a different argument based on the approximation of the function $u_{1}$ (cf. (2.10)) was outlined in [13]. Here the main novelty is that we construct an approximation of that function; an analogous procedure will be applied to other first-order differential operators in the next sections. We also show that conversely any expression of the form $\nabla u(x)+\nabla_{y} u_{1}(x, y)$ is the two-scale limit of $\nabla u_{\varepsilon}$ for some bounded sequence $\left\{u_{\varepsilon}\right\}$ of $W^{1, p}\left(\mathbb{R}^{N}\right)$.

Theorem 2.2. (i) Let $p \in] 1,+\infty\left[\right.$ and $\left\{u_{\varepsilon}\right\}$ be a sequence such that $u_{\varepsilon} \rightarrow u$ in $W^{1, p}\left(\mathbb{R}^{N}\right)$. For any $\varepsilon$ there exists a unique $u_{1 \varepsilon}^{*} \in \ell^{p}\left(W_{*}^{1, p}(\mathcal{Y})\right)$ such that

$$
\begin{gathered}
\int_{\mathcal{Y}}\left[\nabla u_{1 \varepsilon}^{*}(m, y)-\left(\nabla u_{\varepsilon}\right)(\varepsilon(m+y))\right] \cdot \nabla \zeta d y=0 \\
\forall \zeta \in W^{1, p^{\prime}}(\mathcal{Y}), \forall m \in \mathbb{Z}^{N} .
\end{gathered}
$$


Moreover there exists $u_{1} \in L^{p}\left(\mathbb{R}^{N} ; W_{*}^{1, p}(\mathcal{Y})\right)$ such that setting

$$
\begin{aligned}
u_{1 \varepsilon}(\varepsilon(m+y)) & :=u_{1 \varepsilon}^{*}(m, y) \\
z_{\varepsilon}(\varepsilon(m+y)) & :=\nabla u_{1 \varepsilon}^{*}(m, y) \quad \text { for a.a. } y \in Y, \forall m \in \mathbb{Z}^{N},
\end{aligned}
$$

as $\varepsilon \rightarrow 0$ along a suitable subsequence,

$$
u_{1 \varepsilon} \underset{2}{\rightarrow} u_{1} \text { in } L^{p}\left(\mathbb{R}^{N} \times \mathcal{Y}\right), \quad z_{\varepsilon} \underset{2}{\rightarrow} \nabla_{y} u_{1} \text { in } L^{p}\left(\mathbb{R}^{N} \times \mathcal{Y}\right)^{N} .
$$

This entails that, as $\varepsilon \rightarrow 0$ along the extracted subsequence,

$$
\nabla u_{\varepsilon} \underset{2}{\rightarrow} \nabla u+\nabla_{y} u_{1} \quad \text { in } L^{p}\left(\mathbb{R}^{N} \times \mathcal{Y}\right)^{N} .
$$

(ii) Conversely, for any $u \in W^{1, p}\left(\mathbb{R}^{N}\right)$ and any $u_{1} \in L^{p}\left(\mathbb{R}^{N} ; W_{*}^{1, p}(\mathcal{Y})\right)$ there exists a sequence $\left\{u_{\varepsilon}\right\}$ of $W^{1, p}\left(\mathbb{R}^{N}\right)$ such that

$$
u_{\varepsilon} \rightarrow u \quad \text { in } L^{p}\left(\mathbb{R}^{N}\right), \quad \nabla u_{\varepsilon} \underset{2}{\rightarrow} \nabla u+\nabla_{y} u_{1} \quad \text { in } L^{p}\left(\mathbb{R}^{N} \times \mathcal{Y}\right)^{N} .
$$

(Notice that $z_{\varepsilon}$ need not coincide with $\nabla u_{1 \varepsilon}$ a.e. in $\mathbb{R}^{N}$.)

Proof. (i) By Lemma 2.1, $u_{1 \varepsilon}^{*}$ exists and

$$
\left\|z_{\varepsilon}\right\|_{L^{p}\left(\mathbb{R}^{N}\right)^{N}},\left\|u_{1 \varepsilon}\right\|_{L^{p}\left(\mathbb{R}^{N}\right)} \leq \text { Constant (independent of } \varepsilon \text { ). }
$$

By Proposition 1.2 then there exists $u_{1} \in L^{p}\left(\mathbb{R}^{N} \times \mathcal{Y}\right)$ such that, as $\varepsilon \rightarrow 0$ along a suitable subsequence, $u_{1 \varepsilon} \rightarrow u_{1}$ in $L^{p}\left(\mathbb{R}^{N} \times \mathcal{Y}\right)$; hence $u_{1 \varepsilon} \overrightarrow{{ }_{2}} u_{1}$ in $L^{p}\left(\mathbb{R}^{N} \times \mathcal{Y}\right)$. For any $\varphi \in \mathcal{D}\left(\mathbb{R}^{N}\right)$ and $\psi \in \mathcal{D}(\mathcal{Y})^{N}$ we have

$$
\begin{aligned}
\int_{\mathbb{R}^{N}} z_{\varepsilon}(x) \cdot \psi\left(\frac{x}{\varepsilon}\right) \varphi\left(\varepsilon \mathcal{N}\left(\frac{x}{\varepsilon}\right)\right) d x & =\varepsilon^{N} \sum_{m \in \mathbb{Z}^{N}} \int_{\mathcal{Y}} \nabla u_{1 \varepsilon}^{*}(m, y) \cdot \psi(y) \varphi(\varepsilon m) d y \\
& =-\varepsilon^{N} \sum_{m \in \mathbb{Z}^{N}} \int_{\mathcal{Y}} u_{1 \varepsilon}^{*}(m, y) \nabla \cdot \psi(y) \varphi(\varepsilon m) d y \\
& =-\varepsilon^{N} \sum_{m \in \mathbb{Z}^{N}} \int_{\mathcal{Y}} u_{1 \varepsilon}(\varepsilon(m+y)) \nabla \cdot \psi(y) \varphi(\varepsilon m) d y \\
& \rightarrow-\iint_{\mathbb{R}^{N} \times \mathcal{Y}} u_{1}(x, y) \nabla \cdot \psi(y) \varphi(x) d x d y .
\end{aligned}
$$

As $z_{\varepsilon}$ is uniformly bounded in $L^{p}\left(\mathbb{R}^{N}\right)$ and $\varphi\left(\varepsilon \mathcal{N}\left(\frac{x}{\varepsilon}\right)\right) \rightarrow \varphi(x)$ uniformly for $x \in \mathbb{R}^{N}$, the latter convergence also holds if in the first integral $\varphi\left(\varepsilon \mathcal{N}\left(\frac{x}{\varepsilon}\right)\right)$ is replaced by $\varphi(x)$. Thus $u_{1} \in L^{p}\left(\mathbb{R}^{N} ; W_{*}^{1, p}(\mathcal{Y})\right)$ and $(2.9)$ is fulfilled.

(ii) In view of the derivation of (2.10), let us still fix any $\varphi \in \mathcal{D}\left(\mathbb{R}^{N}\right)$ and any $\psi \in \mathcal{D}(\mathcal{Y})^{N}$, and notice that there exists a (unique) $\zeta \in H_{*}^{1}(\mathcal{Y})$ such that

$$
\int_{\mathcal{Y}} \nabla \zeta \cdot \nabla v d y=\int_{\mathcal{Y}} \psi \cdot \nabla v d y \quad \forall v \in H_{*}^{1}(\mathcal{Y}) .
$$


Hence (setting $\nabla \cdot=\operatorname{div}) \Delta \zeta=\nabla \cdot \psi+C$ in $\mathcal{Y}$ for some constant $C \in \mathbb{R}$; integrating over $\mathcal{Y}$, by the $Y$-periodicity of $\zeta$ and $\psi$ we get $C=0$. By the regularity of $\psi$ we infer that $\zeta \in \mathcal{D}(\mathcal{Y})$. By setting $\tilde{\psi}:=\nabla \zeta$ and $\hat{\psi}:=\psi-\tilde{\psi}$ in $\mathcal{Y}$, we thus have

$$
\tilde{\psi}, \hat{\psi} \in \mathcal{D}(\mathcal{Y})^{N}, \quad \nabla \cdot \tilde{\psi}=\nabla \cdot \psi, \quad \nabla \cdot \hat{\psi}=0 \quad \text { in } \mathcal{Y}
$$

By Lemma 1.1, (2.7) and (2.9) we have

$$
\begin{aligned}
\int_{\mathbb{R}^{N}} \nabla u_{\varepsilon}(x) & \cdot \tilde{\psi}\left(\frac{x}{\varepsilon}\right) \varphi\left(\varepsilon \mathcal{N}\left(\frac{x}{\varepsilon}\right)\right) d x \\
& =\int_{\mathbb{R}^{N}} d x \varphi\left(\varepsilon \mathcal{N}\left(\frac{x}{\varepsilon}\right)\right) \int_{\mathcal{Y}}\left(\nabla u_{\varepsilon}\right)\left(\varepsilon \mathcal{N}\left(\frac{x}{\varepsilon}\right)+\varepsilon y\right) \cdot \nabla \zeta(y) d y \\
& =\int_{\mathbb{R}^{N}} d x \varphi\left(\varepsilon \mathcal{N}\left(\frac{x}{\varepsilon}\right)\right) \int_{\mathcal{Y}} \nabla u_{1 \varepsilon}^{*}\left(\mathcal{N}\left(\frac{x}{\varepsilon}\right), y\right) \cdot \nabla \zeta(y) d y \\
& \rightarrow \iint_{\mathbb{R}^{N} \times \mathcal{Y}} \nabla_{y} u_{1}(x, y) \cdot \tilde{\psi}(y) \varphi(x) d x d y .
\end{aligned}
$$

Moreover, as $\nabla \cdot \hat{\psi}=0$ a.e. in $\mathcal{Y}$ and $\int_{\mathcal{Y}} \tilde{\psi}(y) d y=\int_{\mathcal{Y}} \nabla \zeta(y) d y=0$,

$$
\begin{aligned}
\int_{\mathbb{R}^{N}} \nabla u_{\varepsilon}(x) \cdot \hat{\psi}\left(\frac{x}{\varepsilon}\right) \varphi(x) d x & =-\int_{\mathbb{R}^{N}} u_{\varepsilon}(x) \hat{\psi}\left(\frac{x}{\varepsilon}\right) \cdot \nabla \varphi(x) d x \rightarrow \\
-\iint_{\mathbb{R}^{N} \times \mathcal{Y}} u(x) \hat{\psi}(y) \cdot \nabla \varphi(x) d x d y & =\iint_{\mathbb{R}^{N} \times \mathcal{Y}} \nabla u(x) \cdot \hat{\psi}(y) \varphi(x) d x d y \\
\iint_{\mathbb{R}^{N} \times \mathcal{Y}} \nabla_{y} u_{1}(x, y) \cdot \hat{\psi}(y) \varphi(x) d x d y & =-\iint_{\mathbb{R}^{N} \times \mathcal{Y}} u_{1}(x, y) \nabla \cdot \hat{\psi}(y) \varphi(x) d x d y=0, \\
\iint_{\mathbb{R}^{N} \times \mathcal{Y}} \nabla u(x) \cdot \tilde{\psi}(y) \varphi(x) d x d y & =\int_{\mathbb{R}^{N}} \nabla u(x) \varphi(x) d x \cdot \int_{\mathcal{Y}} \tilde{\psi}(y) d y=0 .
\end{aligned}
$$

As $\left\|\nabla u_{\varepsilon}\right\|_{L^{p}\left(\mathbb{R}^{N}\right)^{N}}$ is uniformly bounded and $\varphi\left(\varepsilon \mathcal{N}\left(\frac{x}{\varepsilon}\right)\right) \rightarrow \varphi(x)$ uniformly in $\mathbb{R}^{N}$, cf. (1.1),

$$
\int_{\mathbb{R}^{N}} \nabla u_{\varepsilon}(x) \cdot \psi\left(\frac{x}{\varepsilon}\right) \varphi(x) d x-\int_{\mathbb{R}^{N}} \nabla u_{\varepsilon}(x) \cdot \psi\left(\frac{x}{\varepsilon}\right) \varphi\left(\varepsilon \mathcal{N}\left(\frac{x}{\varepsilon}\right)\right) d x \rightarrow 0 ;
$$

a similar statement holds for the first member of (2.12). Assembling the five latter formulas, we get

$$
\int_{\mathbb{R}^{N}} \nabla u_{\varepsilon}(x) \cdot \psi\left(\frac{x}{\varepsilon}\right) \varphi(x) d x \rightarrow \iint_{\mathbb{R}^{N} \times \mathcal{Y}}\left[\nabla u(x)+\nabla_{y} u_{1}(x, y)\right] \cdot \psi(y) \varphi(x) d x d y
$$

that is $(2.10)$. 
(iii) Let us now come to part (ii) of the thesis. Let us fix any $u \in W^{1, p}\left(\mathbb{R}^{N}\right)$ and any $u_{1} \in L^{p}\left(\mathbb{R}^{N} ; W_{*}^{1, p}(\mathcal{Y})\right)$, define $\rho$ as in (1.6), and set

$$
u_{\varepsilon}(x):=u(x)+\varepsilon \int_{\mathbb{R}^{N}} u_{1}\left(\xi, \frac{x}{\varepsilon}\right) \rho_{\varepsilon}(x-\xi) d \xi \quad \text { for a.a. } x \in \mathbb{R}^{N}
$$

hence $u_{\varepsilon} \rightarrow u$ in $L^{p}\left(\mathbb{R}^{N}\right)$ and

$$
\nabla u_{\varepsilon}(x)=\nabla u(x)+\varepsilon \int_{\mathbb{R}^{N}} u_{1}\left(\xi, \frac{x}{\varepsilon}\right) \nabla \rho_{\varepsilon}(x-\xi) d \xi+\int_{\mathbb{R}^{N}} \nabla_{y} u_{1}\left(\xi, \frac{x}{\varepsilon}\right) \rho_{\varepsilon}(x-\xi) d \xi .
$$

We claim that

$$
\begin{gathered}
\varepsilon \int_{\mathbb{R}^{N}} u_{1}\left(\xi, \frac{x}{\varepsilon}\right) \nabla \rho_{\varepsilon}(x-\xi) d \xi \rightarrow 0 \\
g_{\varepsilon}(x):=\int_{\mathbb{R}^{N}} \nabla_{y} u_{1}\left(\xi, \frac{x}{\varepsilon}\right) \rho_{\varepsilon}(x-\xi) d \xi \underset{2}{\rightarrow} \nabla_{y} u_{1} \quad \text { in } L^{p}\left(\mathbb{R}^{N} \times \mathcal{Y}\right)^{N},
\end{gathered}
$$

which suffices to infer (2.11). As $\varepsilon^{\frac{1}{2}} \int_{\mathbb{R}^{N}} \nabla \rho_{\varepsilon}(x) d x$ is independent of $\varepsilon,(2.14)$ is easily checked. In view of proving (2.15), notice that by Lemma 1.1

$$
\begin{aligned}
\mid g_{\varepsilon}\left(S_{\varepsilon}(x, y)\right) & -\left.\nabla_{y} u_{1}\left(S_{\varepsilon}(x, y), y\right)\right|^{p} \\
& =\left|\int_{\mathbb{R}^{N}} \nabla_{y} u_{1}(\xi, y) \rho_{\varepsilon}\left(S_{\varepsilon}(x, y)-\xi\right) d \xi-\nabla_{y} u_{1}\left(S_{\varepsilon}(x, y), y\right)\right|^{p} \quad \forall \varepsilon,
\end{aligned}
$$

and as $\varepsilon \rightarrow 0$ the latter function vanishes a.e. in $\mathbb{R}^{N} \times \mathcal{Y}$, for $S_{\varepsilon}(x, y) \rightarrow x$ uniformly in $\mathbb{R}^{N} \times \mathcal{Y}$ by (1.2). Thus $g_{\varepsilon} \underset{2}{\rightarrow} \nabla_{y} u_{1}$ a.e. in $\mathbb{R}^{N} \times \mathcal{Y}$. By the equi- $L^{p_{-}}$ integrability of $g_{\varepsilon}$, and by Lemma 2.3 below, we then infer (2.15).

Lemma 2.3 (Two-Scale Vitali's Theorem [35]). Let $p \in\left[1,+\infty\left[,\left\{u_{\varepsilon}\right\}\right.\right.$ be a sequence in $L^{p}\left(\mathbb{R}^{N}\right)$ such that $\sup _{\varepsilon} \int_{\mathbb{R}^{N} \backslash B(0, R)}\left|u_{\varepsilon}(x)\right|^{p} d x \rightarrow 0$ as $R \rightarrow+\infty$, and $u_{\varepsilon} \underset{2}{\rightarrow}$ u a.e. in $\mathbb{R}^{N} \times Y$. Then

$$
u \in L^{p}\left(\mathbb{R}^{N} \times \mathcal{Y}\right), \quad u_{\varepsilon} \underset{2}{\longrightarrow} u \quad \text { in } L^{p}\left(\mathbb{R}^{N} \times \mathcal{Y}\right)
$$

iff $\left\{\left|u_{\varepsilon}\right|^{p}\right\}$ is equi-integrable, in the sense that $\lim _{n \rightarrow \infty} \sup _{\varepsilon} \int_{A_{n}}\left|u_{\varepsilon}(x)\right|^{p} d x=0$ for any sequence $\left\{A_{n}\right\}$ of measurable subsets of $\mathbb{R}^{N}$ such that $A_{n} \searrow \emptyset$.

Remarks. (i) Trivial counterexamples show that the function $u_{1}$ need not be uniquely determined by the sequence $\left\{u_{\varepsilon}\right\}$.

(ii) Denoting the weak one-scale (two-scale, resp.) limit by $\lim _{\varepsilon \rightarrow 0}(1)$ (by $\lim _{\varepsilon \rightarrow 0}{ }^{(2)}$, resp.), (2.10) also reads

$$
\lim _{\varepsilon \rightarrow 0}^{(2)} \nabla u_{\varepsilon}=\lim _{\varepsilon \rightarrow 0}^{(1)} \nabla u_{\varepsilon}+\nabla_{y} u_{1}\left(=\nabla \lim _{\varepsilon \rightarrow 0}^{(1)} u_{\varepsilon}+\nabla_{y} u_{1}\right) \text { a.e. in } \mathbb{R}^{N} \times \mathcal{Y} .
$$

This illustrates the relation between the one-scale and two-scale limit of the gradient. For $p=2$ the decomposition $(2.17)$ is orthogonal in $L^{2}\left(\mathbb{R}^{N} \times \mathcal{Y}\right)^{N}$. 
For $N=1$ the proof of Theorem 2.2 might be simplified, by decomposing $u_{\varepsilon} \rightarrow u$ into its piecewise linear interpolate $\left\{u_{\varepsilon}(m \varepsilon): m \in \mathbb{Z}\right\}$ plus a remainder.

By the argument of Theorem 2.2 one can also study the limit of sequences $\left\{u_{\varepsilon}\right\}$ that are bounded in $L^{p}\left(\mathbb{R}^{N}\right)$ jointly with a fixed directional derivative, $\left\{D_{j} u_{\varepsilon}\right\}$ say. In this case the variables $x_{i}$ with $i \neq j$ are reduced to parameters.

\section{Two-scale convergence of the symmetrized gradient of a vector field}

In this section we assume that $N \in \mathbb{N}, p \in] 1,+\infty[$, and define the symmetrized gradient

$$
\left(\nabla^{s} v\right)_{i j}:=\frac{1}{2}\left(D_{j} v_{i}+D_{i} v_{j}\right) \quad \text { for } i, j=1, \ldots, N, \forall v \in W^{1, p}\left(\mathbb{R}^{N}\right)^{N}
$$

Throughout this paper we shall label spaces of symmetric tensors by the index "s". We also denote by ":" the scalar product in $\mathbb{R}^{N^{2}}$ (i.e. the contraction w.r.t. two indices); thus $A: B:=\sum_{i, j=1}^{N} a_{i j} b_{i j}$ for any $A:=\left\{a_{i j}\right\}, B:=\left\{b_{i j}\right\} \in \mathbb{R}^{N^{2}}$.

The classical Korn inequality (cf. e.g. [17, Sect. VII.2]) can be extended to any $N$. Thus $\left\{v \in L^{p}(\mathcal{Y})^{N}: \nabla^{s} v \in L^{p}(\mathcal{Y})^{N^{2}}\right\}$ equipped with the graph norm coincides with $W^{1, p}(\mathcal{Y})^{N}$. In the Poincaré inequality $\nabla v$ may be replaced by $\nabla^{s} v$, and $W_{*}^{1, p}(\mathcal{Y})^{N}$ may be equipped with the equivalent norm $\left\|\nabla^{s} v\right\|_{L^{p}(\mathcal{Y})^{N^{2}}}$.

Lemma 3.1 (Cell Projection). Let $p \in] 1,+\infty[$ and $\varepsilon>0$. For any $w \in$ $W^{1, p}\left(\mathbb{R}^{N}\right)^{N}$ there exists a unique $w^{*}=w^{*}(m, y) \in \ell^{p}\left(W_{*}^{1, p}(\mathcal{Y})^{N}\right)$ such that

$$
\begin{gathered}
\int_{\mathcal{Y}}\left[\nabla^{s} w^{*}(m, y)-\left(\nabla^{s} w\right)(\varepsilon(m+y))\right]: \nabla^{s} \zeta d y=0 \\
\forall \zeta \in W^{1, p^{\prime}}(\mathcal{Y})^{N}\left(p^{\prime}:=\frac{p}{p-1}\right), \forall m \in \mathbb{Z}^{N} .
\end{gathered}
$$

Moreover there exists a constant $C_{2}>0$ (independent of $w$ and $\varepsilon$ ) such that

$$
\begin{aligned}
& \varepsilon^{\frac{N}{p}}\left\|\nabla^{s} w^{*}\right\|_{\ell^{p}\left(L^{p}(\mathcal{Y})^{N^{2}}\right)}(\left.=\left(\varepsilon^{N} \sum_{m \in \mathbb{Z}^{N}}\left\|\nabla^{s} w^{*}(m, \cdot)\right\|_{L^{p}(\mathcal{Y})^{N^{2}}}^{p}\right)^{\frac{1}{p}}\right) \\
& \leq\left\|\nabla^{s} w\right\|_{L^{p}\left(\mathbb{R}^{N}\right)^{N^{2}}} \\
& \varepsilon^{\frac{N}{p}}\left\|w^{*}\right\|_{\ell^{p}\left(L^{p}(\mathcal{Y})^{N}\right)} \leq C_{2}\left\|\nabla^{s} w\right\|_{L^{p}\left(\mathbb{R}^{N}\right)^{N^{2}}}
\end{aligned}
$$

The argument mimics that of Lemma 2.1, and is omitted. Here the Poincaré inequality is replaced by the Korn inequality. 
Theorem 3.2. (i) Let $\left\{u_{\varepsilon}\right\}$ be a sequence such that $u_{\varepsilon} \rightarrow u$ in $W^{1, p}\left(\mathbb{R}^{N}\right)^{N}$. For any $\varepsilon$ there exists a unique $u_{1 \varepsilon}^{*} \in \ell^{p}\left(W_{*}^{1, p}(\mathcal{Y})^{N}\right)$ such that

$$
\begin{gathered}
\int_{\mathcal{Y}}\left[\nabla^{s} u_{1 \varepsilon}^{*}(m, y)-\left(\nabla^{s} u_{\varepsilon}\right)(\varepsilon(m+y))\right] \cdot \nabla^{s} \zeta d y=0 \\
\forall \zeta \in W^{1, p^{\prime}}(\mathcal{Y})^{N}, \forall m \in \mathbb{Z}^{N} .
\end{gathered}
$$

Moreover there exists $u_{1} \in L^{p}\left(\mathbb{R}^{N} ; W_{*}^{1, p}(\mathcal{Y})^{N}\right)$ such that, setting

$$
\begin{aligned}
u_{1 \varepsilon}(\varepsilon(m+y)) & :=u_{1 \varepsilon}^{*}(m, y) \\
z_{\varepsilon}(\varepsilon(m+y)) & :=\nabla^{s} u_{1 \varepsilon}^{*}(m, y) \quad \text { for a.a. } y \in Y, \forall m \in \mathbb{Z}^{N},
\end{aligned}
$$

as $\varepsilon \rightarrow 0$ along a suitable subsequence

$$
u_{1 \varepsilon} \underset{2}{\rightarrow} u_{1} \quad \text { in } L^{p}\left(\mathbb{R}^{N} \times \mathcal{Y}\right)^{N}, \quad z_{\varepsilon} \underset{2}{\rightarrow} \nabla_{y}^{s} u_{1} \quad \text { in } L^{p}\left(\mathbb{R}^{N} \times \mathcal{Y}\right)^{N^{2}} .
$$

This entails that, as $\varepsilon \rightarrow 0$ along the extracted subsequence,

$$
\nabla^{s} u_{\varepsilon} \underset{2}{\rightarrow} \nabla^{s} u+\nabla_{y}^{s} u_{1} \quad \text { in } L^{p}\left(\mathbb{R}^{N} \times \mathcal{Y}\right)^{N^{2}}
$$

(ii) Conversely, for any $u \in W^{1, p}\left(\mathbb{R}^{N}\right)^{N}$ and any $u_{1} \in L^{p}\left(\mathbb{R}^{N} ; W_{*}^{1, p}(\mathcal{Y})^{N}\right)$ there exists a sequence $\left\{u_{\varepsilon}\right\}$ of $W^{1, p}\left(\mathbb{R}^{N}\right)^{N}$ such that

$$
u_{\varepsilon} \rightarrow u \quad \text { in } L^{p}\left(\mathbb{R}^{N}\right)^{N}, \quad \nabla^{s} u_{\varepsilon} \underset{2}{\rightarrow} \nabla^{s} u+\nabla_{y}^{s} u_{1} \quad \text { in } L^{p}\left(\mathbb{R}^{N} \times \mathcal{Y}\right)^{N^{2}} .
$$

Proof. (i) By Lemma 3.1, $u_{1 \varepsilon}$ exists and

$$
\left\|z_{\varepsilon}\right\|_{L^{p}\left(\mathbb{R}^{N}\right)^{N^{2}}},\left\|u_{1 \varepsilon}\right\|_{L^{p}\left(\mathbb{R}^{N}\right)^{N}} \leq \text { Constant (independent of } \varepsilon \text { ). }
$$

By Proposition 1.2 then there exists $u_{1} \in L^{p}\left(\mathbb{R}^{N} \times \mathcal{Y}\right)^{N}$ such that, as $\varepsilon$ vanishes along a suitable subsequence, $u_{1 \varepsilon} \overrightarrow{2}_{2} u_{1}$ in $L^{p}\left(\mathbb{R}^{N} \times \mathcal{Y}\right)^{N}$. Let us now fix any $\varphi \in \mathcal{D}\left(\mathbb{R}^{N}\right)$ and any $\psi \in \mathcal{D}(\mathcal{Y})_{s}^{N^{2}}$. Setting $(\nabla \cdot \psi)_{i}:=\sum_{j=1}^{N} D_{y_{j}} \psi_{i j}$ for $i=$ $1, \ldots, N$, we have

$$
\begin{aligned}
\int_{\mathbb{R}^{N}} z_{\varepsilon}(x) \cdot \psi\left(\frac{x}{\varepsilon}\right) \varphi\left(\varepsilon \mathcal{N}\left(\frac{x}{\varepsilon}\right)\right) d x & =\varepsilon^{N} \sum_{m \in \mathbb{Z}^{N}} \int_{\mathcal{Y}} \nabla u_{1 \varepsilon}^{*}(m, y): \psi(y) \varphi(\varepsilon m) d y \\
& =-\varepsilon^{N} \sum_{m \in \mathbb{Z}^{N}} \int_{\mathcal{Y}} u_{1 \varepsilon}^{*}(m, y) \cdot \nabla \cdot \psi(y) \varphi(\varepsilon m) d y \\
& =-\varepsilon^{N} \sum_{m \in \mathbb{Z}^{N}} \int_{\mathcal{Y}} u_{1 \varepsilon}(\varepsilon(m+y)) \cdot \nabla \cdot \psi(y) \varphi(\varepsilon m) d y \\
& \rightarrow-\iint_{\mathbb{R}^{N} \times \mathcal{Y}} u_{1}(x, y) \cdot \nabla \cdot \psi(y) \varphi(x) d x d y
\end{aligned}
$$


As $z_{\varepsilon}$ is uniformly bounded in $L^{p}\left(\mathbb{R}^{N}\right)^{N^{2}}$ and $\varphi\left(\varepsilon \mathcal{N}\left(\frac{x}{\varepsilon}\right)\right) \rightarrow \varphi(x)$ uniformly for $x \in \mathbb{R}^{N}$, the latter convergence also holds if in the first integral $\varphi\left(\varepsilon \mathcal{N}\left(\frac{x}{\varepsilon}\right)\right)$ is replaced by $\varphi(x)$. Thus $u_{1} \in L^{p}\left(\mathbb{R}^{N} ; W_{*}^{1, p}(\mathcal{Y})^{N}\right)$ and (3.7) is fulfilled.

(ii) In view of the derivation of (3.8), let us fix any $\varphi \in \mathcal{D}\left(\mathbb{R}^{N}\right)$ and any $\psi \in \mathcal{D}(\mathcal{Y})_{s}^{N^{2}}$, and notice that there exists a (unique) $\zeta \in W_{*}^{1, p}(\mathcal{Y})^{N}$ such that

$$
\int_{\mathcal{Y}} \nabla^{s} \zeta: \nabla^{s} v d y=\int_{\mathcal{Y}} \psi: \nabla^{s} v d y \quad \forall v \in W^{1, p^{\prime}}(\mathcal{Y})^{N}
$$

Setting $\left(\nabla^{s} \cdot \psi\right)_{i}:=\frac{1}{2} \sum_{j=1}^{N}\left(D_{j} \psi_{i j}+D_{j} \psi_{j i}\right)$, we then have $\nabla^{s} \cdot \nabla^{s} \zeta=\nabla^{s} \cdot \psi+C$ in $\mathcal{Y}$ for some constant $C \in \mathbb{R}^{N}$; integrating over $\mathcal{Y}$, by the $Y$-periodicity of $\zeta$ and $\psi$ we get $C=0$. By setting $\tilde{\psi}:=\nabla^{s} \zeta$ and $\hat{\psi}:=\psi-\tilde{\psi}$ in $\mathcal{Y}$, we then have

$$
\tilde{\psi}, \hat{\psi} \in W^{1, p}(\mathcal{Y})^{N^{2}}, \quad \nabla^{s} \cdot \tilde{\psi}=\nabla^{s} \cdot \psi, \quad \nabla^{s} \cdot \hat{\psi}=0 \quad \text { a.e. in } \mathcal{Y} .
$$

By Lemma 1.1, (3.5) and (3.7), we have

$$
\begin{aligned}
\int_{\mathbb{R}^{N}} \nabla^{s} u_{\varepsilon}(x): \tilde{\psi}\left(\frac{x}{\varepsilon}\right) & \varphi\left(\varepsilon \mathcal{N}\left(\frac{x}{\varepsilon}\right)\right) d x \\
& =\int_{\mathbb{R}^{N}} d x \varphi\left(\varepsilon \mathcal{N}\left(\frac{x}{\varepsilon}\right)\right) \int_{\mathcal{Y}} \nabla_{y}^{s} u_{\varepsilon}\left(\varepsilon\left[\mathcal{N}\left(\frac{x}{\varepsilon}\right)+y\right]\right): \nabla^{s} \zeta(y) d y \\
& =\int_{\mathbb{R}^{N}} d x \varphi\left(\varepsilon \mathcal{N}\left(\frac{x}{\varepsilon}\right)\right) \int_{\mathcal{Y}} \nabla^{s} u_{1 \varepsilon}^{*}\left(\mathcal{N}\left(\frac{x}{\varepsilon}\right), y\right): \nabla^{s} \zeta(y) d y \\
& \rightarrow \iint_{\mathbb{R}^{N} \times \mathcal{Y}} \nabla_{y}^{s} u_{1}(x, y): \tilde{\psi}(y) \varphi(x) d x d y
\end{aligned}
$$

Moreover, as $\nabla^{s} \cdot \hat{\psi}=0$ a.e. in $\mathcal{Y}$ and $\int_{\mathcal{Y}} \tilde{\psi}(y) d y=\int_{\mathcal{Y}} \nabla^{s} \zeta(y) d y=0$,

$$
\begin{aligned}
\int_{\mathbb{R}^{N}} \nabla^{s} u_{\varepsilon}(x): \hat{\psi}\left(\frac{x}{\varepsilon}\right) \varphi(x) d x & =-\int_{\mathbb{R}^{N}} u_{\varepsilon}(x) \cdot \hat{\psi}\left(\frac{x}{\varepsilon}\right) \cdot \nabla \varphi(x) d x \rightarrow \\
-\iint_{\mathbb{R}^{N} \times \mathcal{Y}} u(x) \cdot \hat{\psi}(y) \cdot \nabla \varphi(x) d x d y & =\iint_{\mathbb{R}^{N} \times \mathcal{Y}} \nabla^{s} u(x): \hat{\psi}(y) \varphi(x) d x d y, \\
\iint_{\mathbb{R}^{N} \times \mathcal{Y}} \nabla_{y}^{s} u_{1}(x, y): \hat{\psi}(y) \varphi(x) d x d y & =-\iint_{\mathbb{R}^{N} \times \mathcal{Y}} u_{1}(x, y) \cdot \nabla^{s} \cdot \hat{\psi}(y) \varphi(x) d x d y=0, \\
\iint_{\mathbb{R}^{N} \times \mathcal{Y}} \nabla^{s} u(x): \tilde{\psi}(y) \varphi(x) d x d y & =\int_{\mathbb{R}^{N}} \nabla^{s} u(x) \varphi(x) d x: \int_{\mathcal{Y}} \tilde{\psi}(y) d y=0 .
\end{aligned}
$$

As $\left\|\nabla^{s} u_{\varepsilon}\right\|_{L^{p}\left(\mathbb{R}^{N}\right)^{N^{2}}}$ is uniformly bounded and $\varphi\left(\varepsilon \mathcal{N}\left(\frac{x}{\varepsilon}\right)\right) \rightarrow \varphi(x)$ uniformly in $\mathbb{R}^{N}$, cf. (1.1),

$$
\int_{\mathbb{R}^{N}} \nabla^{s} u_{\varepsilon}(x): \psi\left(\frac{x}{\varepsilon}\right) \varphi(x) d x-\int_{\mathbb{R}^{N}} \nabla^{s} u_{\varepsilon}(x): \psi\left(\frac{x}{\varepsilon}\right) \varphi\left(\varepsilon \mathcal{N}\left(\frac{x}{\varepsilon}\right)\right) d x \rightarrow 0 ;
$$


a similar statement holds for the first member of (3.10). By assembling the five latter formulas we end up with

$$
\int_{\mathbb{R}^{N}} \nabla^{s} u_{\varepsilon}(x): \psi\left(\frac{x}{\varepsilon}\right) \varphi(x) d x \rightarrow \iint_{\mathbb{R}^{N} \times \mathcal{Y}}\left[\nabla^{s} u(x)+\nabla_{y}^{s} u_{1}(x, y)\right]: \psi(y) \varphi(x) d x d y
$$

that is (3.8). The proof of part (iii) of the thesis mimics that of Theorem 3.2, and is then omitted.

Remarks. (i) The function $u_{1}$ need not be uniquely determined by the sequence $\left\{u_{\varepsilon}\right\}$.

(ii) Defining $\lim _{\varepsilon \rightarrow 0}(1)$ and $\lim _{\varepsilon \rightarrow 0}(2)$ as in Section 2, (3.8) also reads

$$
\lim _{\varepsilon \rightarrow 0}^{(2)} \nabla^{s} u_{\varepsilon}=\nabla^{s} \lim _{\varepsilon \rightarrow 0}^{(1)} u_{\varepsilon}+\nabla_{y}^{s} u_{1}=\lim _{\varepsilon \rightarrow 0}^{(1)} \nabla^{s} u_{\varepsilon}+\nabla_{y}^{s} u_{1}
$$

a.e. in $\mathbb{R}^{N} \times \mathcal{Y}$. If $p=2$ this decomposition is orthogonal in $L^{2}\left(\mathbb{R}^{N} \times \mathcal{Y}\right)^{N^{2}}$.

\section{Two-scale convergence of the curl of a vector field}

In this section we assume that $N=3$ and $p=2$. We remind the reader that $L_{\text {rot }}^{2}(Y)^{3}:=\left\{v \in L^{2}(Y)^{3}: \nabla \times v \in L^{2}(Y)^{3}\right\}(\nabla \times:=$ curl $)$ is a Hilbert space equipped with the graph norm.

The same applies to $L_{\text {rot }}^{2}\left(\mathbb{R}^{3}\right)^{3}$ and $L_{\text {rot }}^{2}(\mathcal{Y})^{3}$; notice that the tangential components of the functions of the latter space coincide on opposite faces of $Y$.

Lemma 4.1 (Cell Projection). Let $\varepsilon>0$. For any $w \in L_{\text {rot }}^{2}\left(\mathbb{R}^{3}\right)^{3}$ there exists a unique $w^{*}=w^{*}(m, y) \in \ell^{2}\left(H_{*}^{1}(\mathcal{Y})^{3}\right)$ such that for any $m \in \mathbb{Z}^{3}$

$$
\begin{aligned}
& \nabla \cdot w^{*}(m, y)=0 \quad \text { for a. } a . y \in \mathcal{Y} \quad(\nabla \cdot:=\operatorname{div}) \\
& \int_{\mathcal{Y}}\left[\nabla \times w^{*}(m, y)-(\nabla \times w)(\varepsilon(m+y))\right] \cdot \nabla \times \zeta d y=0 \quad \forall \zeta \in H^{1}(\mathcal{Y})^{3} .
\end{aligned}
$$

This entails that there exists a constant $C_{3}>0$ (independent of $w$ and $\varepsilon$ ) such that

$$
\begin{aligned}
\varepsilon^{\frac{3}{2}}\left\|\nabla \times w^{*}\right\|_{\ell^{2}\left(L^{2}(\mathcal{Y})^{3}\right)} & \left.=\left(\varepsilon^{3} \sum_{m \in \mathbb{Z}^{3}}\left\|\nabla \times w^{*}(m, \cdot)\right\|_{L^{2}(\mathcal{Y})^{3}}^{2}\right)^{\frac{1}{2}}\right) \\
& \leq\|\nabla \times w\|_{L^{2}\left(\mathbb{R}^{3}\right)^{3}} \\
\varepsilon^{\frac{3}{2}}\left\|w^{*}\right\|_{\ell^{2}\left(L^{2}(\mathcal{Y})^{3}\right)} & \leq C_{3}\|\nabla \times w\|_{L^{2}\left(\mathbb{R}^{3}\right)^{3}} .
\end{aligned}
$$


Proof. Let us fix any $m \in \mathbb{Z}^{3}$, define the Hilbert space $V_{m}:=\left\{v \in H_{*}^{1}(\mathcal{Y})^{3}\right.$ : $\nabla \cdot v=0\}$, and consider the quadratic functional

$$
V_{m} \rightarrow \mathbb{R}: v \mapsto \int_{\mathcal{Y}}\left(\frac{1}{2}|\nabla \times v|^{2}-(\nabla \times w)(\varepsilon(m+y)) \cdot \nabla \times v\right) d y .
$$

By the Poincaré inequality this functional is coercive on $V_{m}$; hence it has a (unique) minimizer, and this is the unique solution of (4.1). This yields (4.2). It is not restrictive to assume that $w$ is divergence-free, for $w^{*}$ only depends on $\nabla \times w ;(2.7)$ then yields (4.3). The uniqueness of the solution of (4.1) is a simple consequence of (4.3).

Theorem 4.2. (i) Let $\left\{u_{\varepsilon}\right\}$ be a bounded sequence in the space $L_{\mathrm{rot}}^{2}\left(\mathbb{R}^{3}\right)^{3}$ such that $u_{\varepsilon} \overrightarrow{2} u$ in $L^{2}\left(\mathbb{R}^{3} \times \mathcal{Y}\right)^{3}$. For any $\varepsilon$ there exists a unique $u_{1 \varepsilon}^{*} \in \ell^{2}\left(H_{*}^{1}(\mathcal{Y})^{3}\right)$ such that, for any $m \in \mathbb{Z}^{3}$,

$$
\begin{aligned}
& \nabla \cdot u_{1 \varepsilon}^{*}=0 \quad \text { for a.a. } y \in \mathcal{Y} \\
& \int_{\mathcal{Y}}\left[\nabla \times u_{1 \varepsilon}^{*}(m, y)-\left(\nabla \times u_{\varepsilon}\right)(\varepsilon(m+y))\right] \cdot \nabla \times \zeta d y=0 \quad \forall \zeta \in H^{1}(\mathcal{Y})^{3} .
\end{aligned}
$$

Moreover there exists $u_{1} \in L^{2}\left(\mathbb{R}^{3} ; H_{*}^{1}(\mathcal{Y})^{3}\right)$ such that $\nabla_{y} \cdot u_{1}=0$ a.e. in $\mathbb{R}^{3} \times \mathcal{Y}$ and, setting

$$
\begin{aligned}
u_{1 \varepsilon}(\varepsilon(m+y)) & :=u_{1 \varepsilon}^{*}(m, y) \\
z_{\varepsilon}(\varepsilon(m+y)) & :=\nabla \times u_{1 \varepsilon}^{*}(m, y) \quad \text { for a.a. } y \in Y, \forall m \in \mathbb{Z}^{3},
\end{aligned}
$$

as $\varepsilon \rightarrow 0$ along a suitable subsequence

$$
u_{1 \varepsilon} \underset{2}{\rightarrow} u_{1}, \quad z_{\varepsilon} \underset{2}{\overrightarrow{2}} \nabla_{y} \times u_{1} \quad \text { in } L^{2}\left(\mathbb{R}^{3} \times \mathcal{Y}\right)^{3} .
$$

This entails that, as $\varepsilon \rightarrow 0$ along the extracted subsequence, defining $\bar{u}$ as in (1.7),

$$
\nabla \times u_{\varepsilon} \underset{2}{\overrightarrow{2}} \nabla \times \bar{u}+\nabla_{y} \times u_{1} \quad \text { in } L^{2}\left(\mathbb{R}^{3} \times \mathcal{Y}\right)^{3} .
$$

Furthermore $u \in L^{2}\left(\mathbb{R}^{3} ; L_{\text {rot }}^{2}(\mathcal{Y})^{3}\right)$ and for the whole given sequence

$$
\varepsilon \nabla \times u_{\varepsilon} \underset{2}{\rightarrow} \nabla_{y} \times u=0 \quad \text { in } L^{2}\left(\mathbb{R}^{3} \times \mathcal{Y}\right)^{3} .
$$

(ii) Conversely, for any $u \in L^{2}\left(\mathbb{R}^{3} \times \mathcal{Y}\right)^{3}$ such that $\bar{u} \in L_{\text {rot }}^{2}\left(\mathbb{R}^{3}\right)^{3}$ and $\nabla_{y} \times u=0$ in $\mathcal{D}^{\prime}\left(\mathbb{R}^{3} \times \mathcal{Y}\right)^{3}$ and for any $u_{1} \in L^{2}\left(\mathbb{R}^{3} ; H_{*}^{1}(\mathcal{Y})^{3}\right)$, there exists a sequence $\left\{u_{\varepsilon}\right\}$ of $H^{1}\left(\mathbb{R}^{3}\right)^{3}$ such that

$$
u_{\varepsilon} \rightarrow u \quad \text { in } L^{2}\left(\mathbb{R}^{3}\right)^{3}, \quad \nabla \times u_{\varepsilon} \underset{2}{\rightarrow} \nabla \times \bar{u}+\nabla_{y} \times u_{1} \quad \text { in } L^{2}\left(\mathbb{R}^{3} \times \mathcal{Y}\right)^{3} .
$$


Proof. (i) By Lemma 4.1, $u_{1 \varepsilon}$ exists and

$$
\left\|z_{\varepsilon}\right\|_{L^{2}\left(\mathbb{R}^{3}\right)^{3}},\left\|u_{1 \varepsilon}\right\|_{L^{2}\left(\mathbb{R}^{3}\right)^{3}} \leq \text { Constant (independent of } \varepsilon \text { ). }
$$

By Proposition 1.2 then there exists $u_{1} \in L^{2}\left(\mathbb{R}^{3} \times \mathcal{Y}\right)^{3}$ such that, as $\varepsilon \rightarrow 0$ along a suitable subsequence, $u_{1 \varepsilon} \underset{2}{ } u_{1}$ in $L^{2}\left(\mathbb{R}^{3} \times \mathcal{Y}\right)^{3}$. For any $\varphi \in \mathcal{D}\left(\mathbb{R}^{3}\right)$ and any $\psi \in \mathcal{D}(\mathcal{Y})^{3}$ we then have

$$
\begin{aligned}
\int_{\mathbb{R}^{3}} z_{\varepsilon}(x) \cdot \psi\left(\frac{x}{\varepsilon}\right) \varphi\left(\varepsilon \mathcal{N}\left(\frac{x}{\varepsilon}\right)\right) d x & =\varepsilon^{3} \sum_{m \in \mathbb{Z}^{3}} \int_{\mathcal{Y}} \nabla \times u_{1 \varepsilon}^{*}(m, y) \cdot \psi(y) \varphi(\varepsilon m) d y \\
& =-\varepsilon^{3} \sum_{m \in \mathbb{Z}^{3}} \int_{\mathcal{Y}} u_{1 \varepsilon}^{*}(m, y) \cdot \nabla \times \psi(y) \varphi(\varepsilon m) d y \\
& =-\varepsilon^{3} \sum_{m \in \mathbb{Z}^{3}} \int_{\mathcal{Y}} u_{1 \varepsilon}(\varepsilon(m+y)) \cdot \nabla \times \psi(y) \varphi(\varepsilon m) d y \\
& \rightarrow-\iint_{\mathbb{R}^{3} \times \mathcal{Y}} u_{1}(x, y) \cdot \nabla \times \psi(y) \varphi(x) d x d y
\end{aligned}
$$

As $z_{\varepsilon}$ is uniformly bounded in $L^{2}\left(\mathbb{R}^{3}\right)^{3}$ and $\varphi\left(\varepsilon \mathcal{N}\left(\frac{x}{\varepsilon}\right)\right) \rightarrow \varphi(x)$ uniformly for $x \in \mathbb{R}^{3}$, the latter convergence also holds if in the first integral $\varphi\left(\varepsilon \mathcal{N}\left(\frac{x}{\varepsilon}\right)\right)$ is replaced by $\varphi(x)$. Hence $\nabla_{y} \times u_{1} \in L^{2}\left(\mathbb{R}^{3} \times \mathcal{Y}\right)^{3}$ and (4.6) is fulfilled. By $(4.4)_{1}$, we also have $u_{1} \in L^{2}\left(\mathbb{R}^{3} ; H_{*}^{1}(\mathcal{Y})^{3}\right)$ and $\nabla_{y} \cdot u_{1}=0$ a.e. in $\mathbb{R}^{3} \times \mathcal{Y}$.

(ii) Let us fix any $\varphi \in \mathcal{D}\left(\mathbb{R}^{3}\right)$ and any $\psi \in \mathcal{D}(\mathcal{Y})^{3}$. Arguing as for Lemma 4.1 , it is easy to see that there exists $\zeta \in L_{\text {rot* }}^{2}(\mathcal{Y})^{3}$ such that

$$
\int_{\mathcal{Y}}(\nabla \times \zeta-\psi) \cdot \nabla \times v d y=0 \quad \forall v \in H^{1}(\mathcal{Y})^{3}
$$

By setting $\tilde{\psi}:=\nabla \times \zeta$ and $\hat{\psi}:=\psi-\tilde{\psi}$ in $\mathcal{Y}$, we then have $\tilde{\psi}, \hat{\psi} \in L^{2}(\mathcal{Y})^{3}$, $\nabla \times \hat{\psi}=0$ in $\left(L_{\text {rot }}^{2}(\mathcal{Y})^{3}\right)^{\prime}$. By Lemma 1.1, (4.4) and (4.5), we have

$$
\begin{aligned}
\int_{\mathbb{R}^{3}} \nabla \times u_{\varepsilon}(x) & \tilde{\psi}\left(\frac{x}{\varepsilon}\right) \varphi\left(\varepsilon \mathcal{N}\left(\frac{x}{\varepsilon}\right)\right) d x \\
& =\int_{\mathbb{R}^{3}} d x \varphi\left(\varepsilon \mathcal{N}\left(\frac{x}{\varepsilon}\right)\right) \int_{\mathcal{Y}}\left(\nabla \times u_{\varepsilon}\right)\left(\varepsilon\left[\mathcal{N}\left(\frac{x}{\varepsilon}\right)+y\right]\right) \cdot \nabla \times \zeta(y) d y \\
& =\int_{\mathbb{R}^{3}} d x \varphi\left(\varepsilon \mathcal{N}\left(\frac{x}{\varepsilon}\right)\right) \int_{\mathcal{Y}} \nabla \times u_{1 \varepsilon}^{*}\left(\mathcal{N}\left(\frac{x}{\varepsilon}\right), y\right) \cdot \nabla \times \zeta(y) d y \\
& \rightarrow \iint_{\mathbb{R}^{3} \times \mathcal{Y}} \nabla_{y} \times u_{1}(x, y) \cdot \tilde{\psi}(y) \varphi(x) d x d y .
\end{aligned}
$$

(iii) In general $u_{\varepsilon}$ will not converge strongly in $L^{2}\left(\mathbb{R}^{3}\right)^{3}$; we then construct another sequence $\left\{w_{\varepsilon}\right\}$ as follows. Having fixed a test function $\varphi \in \mathcal{D}\left(\mathbb{R}^{3}\right)$, it is 
clear that the limit of $\nabla \times u_{\varepsilon}(x) \cdot \hat{\psi}\left(\frac{x}{\varepsilon}\right) \varphi(x)$ does not depend on the behaviour of $u_{\varepsilon}$ outside the support $S$ of $\varphi$, so that we may assume $S$ to be equal to a ball without loss of generality. Let us then set

$$
V_{S}:=\left\{v \in H^{1}\left(\mathbb{R}^{3}\right)^{3}: v=0 \text { a.e. in } \mathbb{R}^{3} \backslash S, \nabla \cdot v=0\right\} .
$$

For any $\varepsilon>0$, by the Poincaré inequality there exists a (unique) minimizer $w_{\varepsilon} \in V_{S}$ of the functional

$$
\Psi_{\varepsilon}(v):=\int_{\mathbb{R}^{3}}\left(\frac{1}{2}|\nabla \times v|^{2}-\nabla \times u_{\varepsilon} \cdot \nabla \times v\right) d x \quad \forall v \in V_{S} .
$$

We then have

$$
\nabla \cdot w_{\varepsilon}=0, \quad \int_{\mathbb{R}^{3}}\left(\nabla \times w_{\varepsilon}-\nabla \times u_{\varepsilon}\right) \cdot \nabla \times v d x=0 \quad \forall v \in V_{S} .
$$

As $\nabla \times V_{S}=\nabla \times\left\{v \in H^{1}\left(\mathbb{R}^{3}\right)^{3}: v=0\right.$ a.e. in $\left.\mathbb{R}^{3} \backslash S\right\}$, we conclude that

$$
\nabla \times w_{\varepsilon}=\nabla \times u_{\varepsilon} \quad \text { a.e. in } S, \forall \varepsilon .
$$

The sequences $\left\{w_{\varepsilon}\right\}$ is bounded in $H^{1}\left(\mathbb{R}^{3}\right)^{3}$ (at variance with $\left\{u_{\varepsilon}\right\}$ ); hence there exists $w \in H^{1}\left(\mathbb{R}^{3}\right)^{3}$ such that, up to a subsequence,

$$
w_{\varepsilon} \rightarrow w \quad \text { in } H^{1}\left(\mathbb{R}^{3}\right)^{3} .
$$

As $u_{\varepsilon} \rightarrow \bar{u}$ in $L_{\text {rot }}^{2}\left(\mathbb{R}^{3}\right)^{3}$, passing to the weak (one-scale) limit in (4.12) we get

$$
\nabla \times w=\nabla \times \bar{u} \quad \text { a.e. in } S .
$$

(iv) Recalling that $\hat{\psi}$ is curl-free, by the three latter displayed formulas we have

$$
\begin{aligned}
\int_{\mathbb{R}^{3}}\left[\nabla \times u_{\varepsilon}(x)-\nabla \times w_{\varepsilon}(x)\right] \cdot \hat{\psi}\left(\frac{x}{\varepsilon}\right) \varphi(x) d x & =0 \\
\int_{\mathbb{R}^{3}} \nabla \times u_{\varepsilon}(x) \cdot \hat{\psi}\left(\frac{x}{\varepsilon}\right) \varphi(x) d x & =\int_{\mathbb{R}^{3}} \nabla \times w_{\varepsilon}(x) \cdot \hat{\psi}\left(\frac{x}{\varepsilon}\right) \varphi(x) d x \\
& =-\int_{\mathbb{R}^{3}} w_{\varepsilon}(x) \cdot \hat{\psi}\left(\frac{x}{\varepsilon}\right) \times \nabla \varphi(x) d x \rightarrow \\
-\iint_{\mathbb{R}^{3} \times \mathcal{Y}} w(x) \cdot \hat{\psi}(y) \times \nabla \varphi(x) d x d y & =\iint_{\mathbb{R}^{3} \times \mathcal{Y}} \nabla \times w(x) \cdot \hat{\psi}(y) \varphi(x) d x d y \\
& =\iint_{\mathbb{R}^{3} \times \mathcal{Y}} \nabla \times \bar{u}(x) \cdot \hat{\psi}(y) \varphi(x) d x d y \\
\iint_{\mathbb{R}^{3} \times \mathcal{Y}} \nabla_{y} \times u_{1}(x, y) \cdot \hat{\psi}(y) \varphi(x) d x d y & =\iint_{\mathbb{R}^{3} \times \mathcal{Y}} u_{1}(x, y) \cdot \nabla \times \hat{\psi}(y) \varphi(x) d x d y=0 \\
\iint_{\mathbb{R}^{3} \times \mathcal{Y}} \nabla \times \bar{u}(x) \cdot \tilde{\psi}(y) \varphi(x) d x d y & =\iint_{\mathbb{R}^{3}} \nabla \times \bar{u}(x) \varphi(x) d x \cdot \int_{\mathcal{Y}} \tilde{\psi}(y) d y=0 .
\end{aligned}
$$


In (4.11) we may replace $\varphi\left(\varepsilon \mathcal{N}\left(\frac{x}{\varepsilon}\right)\right)$ by $\varphi(x)$, since their difference vanishes uniformly. By this and the three latter displayed formulas above we get

$$
\int_{\mathbb{R}^{3}} \nabla \times u_{\varepsilon}(x) \cdot \psi\left(\frac{x}{\varepsilon}\right) \varphi(x) d x \rightarrow \iint_{\mathbb{R}^{3} \times \mathcal{Y}}\left[\nabla \times \bar{u}(x)+\nabla_{y} \times u_{1}(x, y)\right] \cdot \psi(y) \varphi(x) d x d y,
$$

i.e., (4.7). (4.8) follows by the argument of part (i).

(v) Part (ii) of the thesis can be proved via an argument analogous to that of part (ii) of Theorem 2.2. However here instead of (2.13) we set

$$
u_{\varepsilon}(x):=\bar{u}(x)+\varepsilon \int_{\mathbb{R}^{3}} u_{1}\left(\xi, \frac{x}{\varepsilon}\right) \rho_{\varepsilon}(x-\xi) d \xi \quad \text { for a.a. } x \in \mathbb{R}^{3}
$$

(here with $\bar{u}(x)$ instead of $u(x)$ ). The argument of part (iii) is also analogous to that of Theorem 2.2; however here the strong convergence of the curl does not entail that of the function.

Remarks. (i) The function $u_{1}$ need not be uniquely determined by the sequence $\left\{u_{\varepsilon}\right\}$.

(ii) Defining $\lim _{\varepsilon \rightarrow 0}(1)$ and $\lim _{\varepsilon \rightarrow 0}(2)$ as in Sect. 2, (4.7) also reads

$$
\lim _{\varepsilon \rightarrow 0}^{(2)} \nabla \times u_{\varepsilon}=\nabla \times \lim _{\varepsilon \rightarrow 0}^{(1)} u_{\varepsilon}+\nabla_{y} \times u_{1}=\lim _{\varepsilon \rightarrow 0}^{(1)} \nabla \times u_{\varepsilon}+\nabla_{y} \times u_{1}
$$

a.e. in $\mathbb{R}^{3} \times \mathcal{Y}$. This decomposition is orthogonal in $L^{2}\left(\mathbb{R}^{3} \times \mathcal{Y}\right)^{3}$. Thus

$$
\lim _{\varepsilon \rightarrow 0}^{(2)} \nabla \times u_{\varepsilon} \equiv 0 \quad \Rightarrow \quad \nabla \times \lim _{\varepsilon \rightarrow 0}^{(1)} u_{\varepsilon}=\nabla_{y} \times u_{1} \equiv 0
$$

\section{Two-scale convergence of the divergence of a vector field}

In this section we assume that $N \in \mathbb{N}$ and $p=2$. It is known that $L_{\text {div }}^{2}(Y)^{N}:=$ $\left\{v \in L^{2}(Y)^{N}: \nabla \cdot v \in L^{2}(Y)\right\}(\nabla \cdot:=\operatorname{div})$ is a Hilbert space equipped with the graph norm. We similarly define $L_{\text {div }}^{2}\left(\mathbb{R}^{N}\right)^{N}$ and $L_{\text {div }}^{2}(\mathcal{Y})^{N}$; notice that the normal traces of the functions of the latter space coincide on opposite faces of $Y$. Dealing with $N \neq 3$, we define the curl as the antisymmetric part of the Hessian matrix:

$$
(\nabla \times v)_{i j}:=D_{j} v_{i}-D_{i} v_{j} \quad \forall i, j \in\{1, \ldots, N\}, \forall v \in \mathcal{D}^{\prime}\left(\mathbb{R}^{N}\right)^{N} .
$$

Lemma 5.1 (Cell Projection). Let $\varepsilon>0$. For any $w \in L_{\mathrm{div}}^{2}\left(\mathbb{R}^{N}\right)^{N}$ there exists a unique $w^{*}=w^{*}(m, y) \in \ell^{2}\left(H_{*}^{1}(\mathcal{Y})^{N}\right)$ such that for any $m \in \mathbb{Z}^{N}$

$$
\begin{aligned}
& \nabla \times w^{*}(m, y)=0 \quad \text { for a.a. } y \in \mathcal{Y} \\
& \int_{\mathcal{Y}}\left[\nabla \cdot w^{*}(m, y)-(\nabla \cdot w)(\varepsilon(m+y))\right] \eta d y=0 \quad \forall \eta \in L_{*}^{2}(\mathcal{Y}) .
\end{aligned}
$$


This entails that there exists a constant $C_{4}>0$ (independent of $w$ and $\varepsilon$ ) such that

$$
\begin{aligned}
\varepsilon^{\frac{N}{2}}\left\|\nabla \cdot w^{*}\right\|_{\ell^{2}\left(L^{2}(\mathcal{Y})\right)} & \left.=\left(\varepsilon^{N} \sum_{m \in \mathbb{Z}^{N}}\left\|\nabla \cdot w^{*}(m, \cdot)\right\|_{L^{2}(\mathcal{Y})}^{2}\right)^{\frac{1}{2}}\right) \\
& \leq\|\nabla \cdot w\|_{L^{2}\left(\mathbb{R}^{N}\right)} \\
\varepsilon^{\frac{N}{2}}\left\|w^{*}\right\|_{\ell^{2}\left(L^{2}(\mathcal{Y})^{N}\right)} & \leq C_{4}\|\nabla \cdot w\|_{L^{2}\left(\mathbb{R}^{N}\right)} .
\end{aligned}
$$

Proof. An argument analogous to that of Lemma 4.1, with the divergence in place of the curl operator, yields the existence and uniqueness of $w^{*}$.

It is not restrictive to assume that $w$ is curl-free, for $w_{\varepsilon}$ only depends on $\nabla \cdot w$; hence $\int_{\mathcal{Y}}|\nabla w|^{2} d x=\int_{\mathcal{Y}}|\nabla \cdot w|^{2} d x$, and (5.4) follows from (2.3).

Theorem 5.2. (i) Let $\left\{u_{\varepsilon}\right\}$ be a bounded sequence in $L_{\text {div }}^{2}\left(\mathbb{R}^{N}\right)^{N}$ such that $u_{\varepsilon} \underset{2}{\longrightarrow} u$ in $L^{2}\left(\mathbb{R}^{N} \times \mathcal{Y}\right)^{N}$. For any $\varepsilon$ there exists a unique $u_{1 \varepsilon}^{*} \in \ell^{2}\left(H_{*}^{1}(\mathcal{Y})^{N}\right)^{N}$ such that, for any $m \in \mathbb{Z}^{N}$,

$$
\begin{aligned}
& \nabla \times u_{1 \varepsilon}^{*}(m, y)=0 \quad \text { for a.a. } y \in \mathcal{Y} \\
& \int_{\mathcal{Y}}\left[\nabla \cdot u_{1 \varepsilon}^{*}(m, y)-(\nabla \cdot w)(\varepsilon(m+y))\right] \eta d y=0 \quad \forall \eta \in L_{*}^{2}(\mathcal{Y}) .
\end{aligned}
$$

Moreover there exists $u_{1} \in L^{2}\left(\mathbb{R}^{N} ; H_{*}^{1}(\mathcal{Y})^{N}\right)$ such that $\nabla_{y} \times u_{1}=0$ a.e. in $\mathbb{R}^{N}$, and, setting

$$
\begin{aligned}
u_{1 \varepsilon}(\varepsilon(m+y)) & :=u_{1 \varepsilon}^{*}(m, y) \\
z_{\varepsilon}(\varepsilon(m+y)) & :=\nabla \cdot u_{1 \varepsilon}^{*}(m, y) \quad \text { for a.a. } y \in Y, \forall m \in \mathbb{Z}^{N}
\end{aligned}
$$

as $\varepsilon \rightarrow 0$ along a suitable subsequence

$$
u_{1 \varepsilon} \underset{2}{\longrightarrow} u_{1} \text { in } L^{2}\left(\mathbb{R}^{N} \times \mathcal{Y}\right)^{N}, \quad z_{\varepsilon} \underset{2}{\longrightarrow} \nabla_{y} \cdot u_{1} \text { in } L^{2}\left(\mathbb{R}^{N} \times \mathcal{Y}\right) .
$$

This entails that, as $\varepsilon \rightarrow 0$ along the extracted subsequence, defining $\bar{u}$ as in (1.7),

$$
\nabla \cdot u_{\varepsilon} \underset{2}{\rightarrow} \nabla \cdot \bar{u}+\nabla_{y} \cdot u_{1} \quad \text { in } L^{2}\left(\mathbb{R}^{N} \times \mathcal{Y}\right) .
$$

Furthermore $u \in L^{2}\left(\mathbb{R}^{N} ; L_{\mathrm{div}}^{2}(\mathcal{Y})^{N}\right)$ and for the whole given sequence

$$
\varepsilon \nabla \cdot u_{\varepsilon} \underset{2}{\longrightarrow} \nabla_{y} \cdot u=0 \quad \text { in } L^{2}\left(\mathbb{R}^{N} \times \mathcal{Y}\right)
$$

(ii) Conversely, for any $u \in L^{2}\left(\mathbb{R}^{N} \times \mathcal{Y}\right)^{N}$ such that $\bar{u} \in L_{\text {div }}^{2}\left(\mathbb{R}^{N}\right)^{N}$ and $\nabla_{y} \cdot u=0$ in $\mathcal{D}^{\prime}\left(\mathbb{R}^{N} \times \mathcal{Y}\right)$ and for any $u_{1} \in L^{2}\left(\mathbb{R}^{N} ; H_{*}^{1}(\mathcal{Y})^{N}\right)$, there exists a sequence $\left\{u_{\varepsilon}\right\}$ of $H^{1}\left(\mathbb{R}^{N}\right)^{N}$ such that

$$
u_{\varepsilon} \rightarrow u \quad \text { in } L^{2}\left(\mathbb{R}^{N}\right)^{N}, \quad \nabla \cdot u_{\varepsilon} \underset{2}{\rightarrow} \nabla \cdot \bar{u}+\nabla_{y} \cdot u_{1} \quad \text { in } L^{2}\left(\mathbb{R}^{N} \times \mathcal{Y}\right) .
$$


Proof. (i) By Lemma 5.1, $u_{1 \varepsilon}$ exists and

$$
\left\|z_{\varepsilon}\right\|_{L^{2}\left(\mathbb{R}^{N}\right)},\left\|u_{1 \varepsilon}\right\|_{L^{2}\left(\mathbb{R}^{N}\right)^{N}} \leq \text { Constant (independent of } \varepsilon \text { ). }
$$

By Proposition 1.2 then there exists $u_{1} \in L^{2}\left(\mathbb{R}^{N} \times \mathcal{Y}\right)^{N}$ such that, as $\varepsilon \rightarrow 0$ along a suitable subsequence, $u_{1 \varepsilon} \underset{2}{ } u_{1}$ in $L^{2}\left(\mathbb{R}^{N} \times \mathcal{Y}\right)^{N}$. For any $\varphi \in \mathcal{D}\left(\mathbb{R}^{N}\right)$ and any $\psi \in \mathcal{D}(\mathcal{Y})$, we have

$$
\begin{aligned}
\int_{\mathbb{R}^{N}} z_{\varepsilon}(x) \psi\left(\frac{x}{\varepsilon}\right) \varphi\left(\varepsilon \mathcal{N}\left(\frac{x}{\varepsilon}\right)\right) d x & =\varepsilon^{N} \sum_{m \in \mathbb{Z}^{N}} \int_{\mathcal{Y}} \nabla \cdot u_{1 \varepsilon}^{*}(m, y) \psi(y) \varphi(\varepsilon m) d y \\
& =-\varepsilon^{N} \sum_{m \in \mathbb{Z}^{N}} \int_{\mathcal{Y}} u_{1 \varepsilon}^{*}(m, y) \cdot \nabla \psi(y) \varphi(\varepsilon m) d y \\
& =-\varepsilon^{N} \sum_{m \in \mathbb{Z}^{N}} \int_{\mathcal{Y}} u_{1 \varepsilon}(\varepsilon(m+y)) \cdot \nabla \psi(y) \varphi(\varepsilon m) d y \\
& \rightarrow-\iint_{\mathbb{R}^{N \times \mathcal{Y}}} u_{1}(x, y) \cdot \nabla \psi(y) \varphi(x) d x d y
\end{aligned}
$$

As $z_{\varepsilon}$ is uniformly bounded in $L^{2}\left(\mathbb{R}^{N}\right)^{N}$ and $\varphi\left(\varepsilon \mathcal{N}\left(\frac{x}{\varepsilon}\right)\right) \rightarrow \varphi(x)$ uniformly for $x \in \mathbb{R}^{N}$, the latter convergence also holds if in the first integral $\varphi\left(\varepsilon \mathcal{N}\left(\frac{x}{\varepsilon}\right)\right)$ is replaced by $\varphi(x)$. Moreover $\nabla_{y} \times u_{1}=0$ a.e. in $\mathbb{R}^{N} \times \mathcal{Y}$, by $(5.5)_{1}$. Thus $u_{1} \in L^{2}\left(\mathbb{R}^{N} ; H_{*}^{1}(\mathcal{Y})^{N}\right)$ and (5.7) is fulfilled.

(ii) Let us fix any $\varphi \in \mathcal{D}\left(\mathbb{R}^{N}\right)$, any $\psi \in \mathcal{D}(\mathcal{Y})$, and define $\bar{\psi}$ and $\tilde{\psi}$ as in (1.7). By (5.5) and (5.7),

$$
\begin{aligned}
\int_{\mathbb{R}^{N}} \nabla \cdot u_{\varepsilon} \tilde{\psi}\left(\frac{x}{\varepsilon}\right) \varphi\left(\varepsilon \mathcal{N}\left(\frac{x}{\varepsilon}\right)\right) d x & =\int_{\mathbb{R}^{N}} \varphi\left(\varepsilon \mathcal{N}\left(\frac{x}{\varepsilon}\right)\right) d x \int_{\mathcal{Y}} \nabla \cdot u_{\varepsilon}\left(\varepsilon \mathcal{N}\left(\frac{x}{\varepsilon}\right)+\varepsilon y\right) \tilde{\psi}\left(\frac{x}{\varepsilon}\right) d y \\
& =\int_{\mathbb{R}^{N}} \varphi\left(\varepsilon \mathcal{N}\left(\frac{x}{\varepsilon}\right)\right) d x \int_{\mathcal{Y}} \nabla \cdot u_{1 \varepsilon}^{*}\left(\mathcal{N}\left(\frac{x}{\varepsilon}\right), y\right) \tilde{\psi}\left(\frac{x}{\varepsilon}\right) d y \\
& \rightarrow \iint_{\mathbb{R}^{N} \times \mathcal{Y}} \nabla_{y} \cdot u_{1}(x, y) \tilde{\psi}(y) \varphi(x) d x d y
\end{aligned}
$$

As $u_{\varepsilon} \rightarrow \bar{u}$ weakly in $L^{2}\left(\mathbb{R}^{N}\right)^{N}$,

$$
\begin{aligned}
\int_{\mathbb{R}^{N}} \nabla \cdot u_{\varepsilon}(x) \bar{\psi} \varphi(x) d x & =-\int_{\mathbb{R}^{N}} u_{\varepsilon}(x) \bar{\psi} \cdot \nabla \varphi(x) d x \rightarrow \\
-\int_{\mathbb{R}^{N}} \bar{u}(x) \bar{\psi} \cdot \nabla \varphi(x) d x d y & =\iint_{\mathbb{R}^{N} \times \mathcal{Y}} \nabla \cdot \bar{u}(x) \bar{\psi} \varphi(x) d x d y .
\end{aligned}
$$

Moreover, as $\int_{\mathcal{Y}} \nabla_{y} \cdot u_{1}(x, y) d y=0$ for a.a. $x$ and $\int_{\mathcal{Y}} \tilde{\psi}(y) d y=0$,

$$
\begin{aligned}
\iint_{\mathbb{R}^{N} \times \mathcal{Y}} \nabla_{y} \cdot u_{1}(x, y) \bar{\psi} \varphi(x) d x d y & =\int_{\mathcal{Y}} \nabla_{y} \cdot u_{1}(x, y) d y \int_{\mathbb{R}^{N}} \bar{\psi} \varphi(x) d x=0 \\
\iint_{\mathbb{R}^{N} \times \mathcal{Y}} \nabla \cdot \bar{u}(x) \tilde{\psi}(y) \varphi(x) d x d y & =\int_{\mathbb{R}^{N}} \nabla \cdot \bar{u}(x) \varphi(x) d x \int_{\mathcal{Y}} \tilde{\psi}(y) d y=0 .
\end{aligned}
$$


As $\varphi\left(\varepsilon \mathcal{N}\left(\frac{x}{\varepsilon}\right)\right) \rightarrow \varphi(x)$ uniformly in $\mathbb{R}^{N}$, the four latter formulas yield

$$
\int_{\mathbb{R}^{N}} \nabla \cdot u_{\varepsilon}(x) \psi\left(\frac{x}{\varepsilon}\right) \varphi(x) d x \rightarrow \iint_{\mathbb{R}^{N} \times \mathcal{Y}}\left[\nabla \cdot \bar{u}(x)+\nabla_{y} \cdot u_{1}(x, y)\right] \psi(y) \varphi(x) d x d y,
$$

i.e., (5.8). (5.9) follows by the argument of part (i). Parts (ii) can be proved as in Theorems 2.2 and 4.2 , defining $u_{\varepsilon}$ as in (4.13).

Remarks. (i) The function $u_{1}$ need not be uniquely determined by the sequence $\left\{u_{\varepsilon}\right\}$.

(ii) Defining $\lim _{\varepsilon \rightarrow 0}(1)$ and $\lim _{\varepsilon \rightarrow 0}(2)$ as in Sect. 2, (5.8) also reads

$$
\lim _{\varepsilon \rightarrow 0}^{(2)} \nabla \cdot u_{\varepsilon}=\nabla \cdot \lim _{\varepsilon \rightarrow 0}^{(1)} u_{\varepsilon}+\nabla_{y} \cdot u_{1}=\lim _{\varepsilon \rightarrow 0}^{(1)} \nabla \cdot u_{\varepsilon}+\nabla_{y} \cdot u_{1}
$$

a.e. in $\mathbb{R}^{N} \times \mathcal{Y}$. This decomposition is orthogonal in $L^{2}\left(\mathbb{R}^{N} \times \mathcal{Y}\right)$. In particular if $\nabla \cdot u_{\varepsilon} \underset{2}{2} 0$ in $L^{2}\left(\mathbb{R}^{N} \times \mathcal{Y}\right)$ then $\nabla \cdot \bar{u}=\nabla_{y} \cdot u_{1} \equiv 0$, consistently with part (iii) of Proposition 1.14 of [1].

\section{Two-scale convergence of the divergence of a tensor field}

In this section we assume that $N=3, p=2$, and define the divergence and the curl of a $3 \times 3$-tensor field $v \in L^{2}(Y)^{9}$ as follows, setting $v_{i}:=\left(v_{i 1}, v_{i 2}, v_{i 3}\right)$ :

$$
(\nabla \cdot v)_{i}:=\nabla \cdot\left(v_{i}\right), \quad(\nabla \times v)_{i}:=\nabla \times\left(v_{i}\right) \quad \text { in } \mathcal{D}^{\prime}(Y)^{3}, \text { for } i=1,2,3 .
$$

Notice that $\|\nabla \times v\|_{L^{2}(Y)^{9}}^{2}+\|\nabla \cdot v\|_{L^{2}(Y)^{3}}^{2}=\|\nabla v\|_{L^{2}(Y)^{27}}^{2}$ for any $v \in H^{1}(Y)^{9}$.

We also set $L_{\operatorname{div}}^{2}(Y)^{9}:=\left\{v \in L^{2}(Y)^{9}: \nabla \cdot v \in L^{2}(Y)^{3}\right\}$; this is a Hilbert subspace of $H^{1}(Y)^{9}$ equipped with the graph norm. We similarly define $L_{\text {div }}^{2}\left(\mathbb{R}^{3}\right)^{9}$, $L_{\operatorname{div}}^{2}(\mathcal{Y})^{9}$, and so on.

Theorem 6.1. (i) Let $\left\{u_{\varepsilon}\right\}$ be a bounded sequence of $L_{\mathrm{div}}^{2}\left(\mathbb{R}^{3}\right)^{9}$ such that $u_{\varepsilon} \overrightarrow{2} u$ in $L^{2}\left(\mathbb{R}^{3} \times \mathcal{Y}\right)^{9}$. For any $\varepsilon$ there exists a unique $u_{1 \varepsilon}^{*} \in \ell^{2}\left(H_{*}^{1}(\mathcal{Y})^{9}\right)$ such that, for any $m \in \mathbb{Z}^{3}$,

$$
\begin{aligned}
& \nabla \times u_{1 \varepsilon}^{*}(m, y)=0 \quad \text { for a.a. } y \in \mathcal{Y} \\
& \int_{\mathcal{Y}}\left[\nabla \cdot u_{1 \varepsilon}^{*}(m, y)-\left(\nabla \cdot u_{\varepsilon}\right)(\varepsilon(m+y))\right] \eta d y=0 \quad \forall \eta \in L_{*}^{2}(\mathcal{Y}) .
\end{aligned}
$$

Moreover there exists $u_{1} \in L^{2}\left(\mathbb{R}^{3} ; H_{*}^{1}(\mathcal{Y})^{9}\right)$ such that $\nabla_{y} \times u_{1}=0$ a.e. in $\mathcal{Y}$ for a.a. $x \in \mathbb{R}^{3}$, and, setting

$$
\begin{aligned}
u_{1 \varepsilon}(\varepsilon(m+y)) & :=u_{1 \varepsilon}^{*}(m, y) \\
z_{\varepsilon}(\varepsilon(m+y)) & :=\nabla \cdot u_{1 \varepsilon}^{*}(m, y) \quad \text { for a.a. } y \in Y, \forall m \in \mathbb{Z}^{3},
\end{aligned}
$$


as $\varepsilon \rightarrow 0$ along a suitable subsequence

$$
u_{1 \varepsilon} \underset{2}{\rightarrow} u_{1} \text { in } L^{2}\left(\mathbb{R}^{3} \times \mathcal{Y}\right)^{9}, \quad z_{\varepsilon} \underset{2}{\overrightarrow{2}} \nabla_{y} \cdot u_{1} \text { in } L^{2}\left(\mathbb{R}^{3} \times \mathcal{Y}\right)^{3} .
$$

This entails that, as $\varepsilon \rightarrow 0$ along the extracted subsequence, defining $\bar{u}$ as in $(1.7)$,

$$
\nabla \cdot u_{\varepsilon} \underset{2}{\rightarrow} \nabla \cdot \bar{u}+\nabla_{y} \cdot u_{1} \quad \text { in } L^{2}\left(\mathbb{R}^{3} \times \mathcal{Y}\right)^{3} .
$$

Furthermore $u \in L^{2}\left(\mathbb{R}^{3} ; L_{\mathrm{div}}^{2}(\mathcal{Y})^{9}\right)$ and for the whole given sequence

$$
\varepsilon \nabla \cdot u_{\varepsilon} \underset{2}{\rightarrow} \nabla_{y} \cdot u=0 \quad \text { in } L^{2}\left(\mathbb{R}^{3} \times \mathcal{Y}\right)^{3} .
$$

If the tensors $u_{\varepsilon}$ are symmetric, then $u_{1}$ is also symmetric.

(ii) Conversely, for any $u \in L^{2}\left(\mathbb{R}^{3} \times \mathcal{Y}\right)^{9}$ such that $\bar{u} \in L_{\text {div }}^{2}\left(\mathbb{R}^{3}\right)^{9}$ and $\nabla_{y} \cdot u=0$ in $\mathcal{D}^{\prime}\left(\mathbb{R}^{3} \times \mathcal{Y}\right)^{3}$ and for any $u_{1} \in L^{2}\left(\mathbb{R}^{3} ; H_{*}^{1}(\mathcal{Y})^{9}\right)$, there exists a sequence $\left\{u_{\varepsilon}\right\}$ of $H^{1}\left(\mathbb{R}^{3}\right)^{9}$ such that

$$
u_{\varepsilon} \rightarrow u \quad \text { in } L^{2}\left(\mathbb{R}^{3}\right)^{9}, \quad \nabla \cdot u_{\varepsilon} \underset{2}{\rightarrow} \nabla \cdot \bar{u}+\nabla_{y} \cdot u_{1} \quad \text { in } L^{2}\left(\mathbb{R}^{3} \times \mathcal{Y}\right)^{3} .
$$

Proof. It suffices to apply Theorem 5.2 to the sequences of vectors $u_{\varepsilon i}:=$ $\left(u_{\varepsilon i 1}, u_{\varepsilon i 2}, u_{\varepsilon i 3}\right)$, for $i=1,2,3$. Part (ii) can also be proved via the procedure of Theorems 2.2 and 4.2, defining $u_{\varepsilon}$ as in (4.13).

Remarks. (i) The function $u_{1}$ need not be uniquely determined by the sequence $\left\{u_{\varepsilon}\right\}$.

(ii) Defining $\lim _{\varepsilon \rightarrow 0}(1)$ and $\lim _{\varepsilon \rightarrow 0}(2)$ as in Sect. 2, (6.5) also reads

$$
\lim _{\varepsilon \rightarrow 0}^{(2)} \nabla \cdot u_{\varepsilon}=\nabla \cdot \lim _{\varepsilon \rightarrow 0}{ }^{(1)} u_{\varepsilon}+\nabla_{y} \cdot u_{1}=\lim _{\varepsilon \rightarrow 0}{ }^{(1)} \nabla \cdot u_{\varepsilon}+\nabla_{y} \cdot u_{1}
$$

a.e. in $\mathbb{R}^{3} \times \mathcal{Y}$. This decomposition is orthogonal in $L^{2}\left(\mathbb{R}^{3} \times \mathcal{Y}\right)^{3}$.

\section{Two-scale limit of some classical laws}

In this section we illustrate some simple applications of the above results, in view of the application to the homogenization of linear and nonlinear problems in electromagnetism and continuum mechanics, cf. e.g. [36]-[39].

Two-scale limit of the Ampère law. Let us consider the classical Ampère law of magnetostatics, and assume that the magnetic field $H$ and the electric current field $J$ depend on a scale parameter $\varepsilon$ :

$$
\nabla \times H_{\varepsilon}=4 \pi J_{\varepsilon} \quad \text { a.e. in } \mathbb{R}^{3} .
$$


Under boundedness hypotheses (here omitted), after Theorem 4.2 as $\varepsilon$ vanishes along a suitable sequence

$$
J_{\varepsilon} \underset{2}{\overrightarrow{2}} J, \quad H_{\varepsilon} \underset{2}{\longrightarrow} H, \quad \nabla \times H_{\varepsilon} \underset{2}{\overrightarrow{2}} \nabla \times \bar{H}+\nabla_{y} \times H_{1} \quad \text { in } L^{2}\left(\mathbb{R}^{3} \times \mathcal{Y}\right)^{3} .
$$

Defining $\bar{J}$ and $\tilde{J}$ as in (1.7), we then get the coarse- and fine-scale Ampère laws:

$$
\begin{aligned}
\nabla \times \bar{H} & =4 \pi \bar{J} & & \text { a.e. in } \mathbb{R}^{3}, \\
\nabla_{y} \times H & =0, \quad \nabla_{y} \times H_{1}=4 \pi \tilde{J} & & \text { a.e. in } \mathbb{R}^{3} \times \mathcal{Y} .
\end{aligned}
$$

Moreover, as $J_{\varepsilon}$ is divergence-free, by Theorem 5.2 ,

$$
\nabla \cdot \bar{J}=0 \quad \text { a.e. in } \mathbb{R}^{3}, \quad \nabla_{y} \cdot \tilde{J}=0 \quad \text { a.e. in } \mathbb{R}^{3} \times \mathcal{Y} .
$$

Two-scale limit of the Gauss law. Next we consider the classical Gauss law of electrostatics, and assume that the electric displacement $D$ and the electric charge $\gamma$ depend on a parameter $\varepsilon$ :

$$
\nabla \cdot D_{\varepsilon}=4 \pi \gamma_{\varepsilon} \quad \text { a.e. in } \mathbb{R}^{3} .
$$

Under boundedness hypotheses, after Theorem 5.2 as $\varepsilon$ vanishes along a suitable sequence

$$
\begin{aligned}
& D_{\varepsilon} \underset{2}{\rightarrow} D \quad \text { in } L^{2}\left(\mathbb{R}^{3} \times \mathcal{Y}\right)^{3} \\
& \gamma_{\varepsilon} \underset{2}{\rightarrow} \gamma, \quad \nabla \cdot D_{\varepsilon} \underset{2}{\longrightarrow} \nabla \cdot \bar{D}+\nabla_{y} \cdot D_{1} \quad \text { in } L^{2}\left(\mathbb{R}^{3} \times \mathcal{Y}\right) \text {. }
\end{aligned}
$$

Defining $\bar{\gamma}$ and $\tilde{\gamma}$ as in (1.7), we then get the coarse- and fine-scale Gauss laws:

$$
\begin{aligned}
\nabla \cdot \bar{D} & =4 \pi \bar{\gamma} & & \text { a.e. in } \mathbb{R}^{3} \\
\nabla_{y} \cdot D & =0, \quad \nabla_{y} \cdot D_{1}=4 \pi \tilde{\gamma} & & \text { a.e. in } \mathbb{R}^{3} \times \mathcal{Y} .
\end{aligned}
$$

Two-scale limit of the system of linear elasticity. Let us denote the displacement by $u$, the deformation tensor by $e$, the Cauchy stress by $\sigma$, and an applied load by $f$ in a domain $\Omega$ of $\mathbb{R}^{3}$. Assuming dependence on a scale parameter $\varepsilon$, the definition of $e$ and the balance of momentum read

$$
\nabla^{s} u_{\varepsilon}=e_{\varepsilon}, \quad \nabla \cdot \sigma_{\varepsilon}=f \quad \text { a.e. in } \Omega .
$$

(Of course a further equation is needed to close the problem). Under suitable boundedness hypotheses, after Theorems 3.2 and 6.2 as $\varepsilon$ vanishes along a suitable sequence

$$
\begin{aligned}
& u_{\varepsilon} \rightarrow u \quad \text { in } W^{1, p}\left(\mathbb{R}^{3}\right)^{3}, \quad e_{\varepsilon} \underset{2}{\rightarrow} e \quad \text { in } L^{p}\left(\mathbb{R}^{3} \times \mathcal{Y}\right)^{9} \quad(p \in] 1,+\infty[), \\
& \sigma_{\varepsilon} \underset{2}{\overrightarrow{2}} \sigma \quad \text { in } L^{2}\left(\mathbb{R}^{3} \times \mathcal{Y}\right)^{9}, \quad \nabla \cdot \sigma_{\varepsilon} \underset{2}{\overrightarrow{2}} \nabla \cdot \bar{\sigma}+\nabla_{y} \cdot \sigma_{1} \quad \text { in } L^{2}\left(\mathbb{R}^{3} \times \mathcal{Y}\right)^{3} \text {. }
\end{aligned}
$$


Notice that $u$ is independent of the fine-scale variable $y$, cf. Theorem 2.2. Defining $\bar{e}, \tilde{e}$ and $\bar{f}, \tilde{f}$ as in (1.7), we then get the coarse- and fine-scale laws:

$$
\begin{aligned}
\nabla^{s} u & =\bar{e}, & \nabla \cdot \bar{\sigma} & =\bar{f} & & \text { a.e. in } \mathbb{R}^{3} \\
\nabla_{y}^{s} u_{1} & =\tilde{e}, & \nabla_{y} \cdot \sigma & =0, \quad \nabla_{y} \cdot \sigma_{1}=\tilde{f} & & \text { a.e. in } \mathbb{R}^{3} \times \mathcal{Y} .
\end{aligned}
$$

\section{On the homogenization of some elliptic equations}

So far we confined ourselves to first-order linear differential operators with constant coefficients. It is clear that several results take over to operators with $x$-dependent coefficients. One may also include coefficients that also depend on the unknown function $u_{\varepsilon}$, provided that the latter converges strongly two-scale.

The results that we derived so far are based on the $L^{2}$-boundedness of some first-order operators, and may be applied to the homogenization of a large number of partial differential equations. Here we illustrate a simple example.

A quasilinear elliptic equation. We deal with the system

$$
\begin{aligned}
& \nabla \times\left[A\left(u_{\varepsilon}(x), x, \frac{x}{\varepsilon}\right) \cdot\left(\nabla \times u_{\varepsilon}+f\right)\right]=0 \quad \text { in } \mathbb{R}^{3} . \\
& \nabla \cdot u_{\varepsilon}=0
\end{aligned}
$$

This equation may account for equilibrium in a heterogeneous and anisotropic electric conductor that occupies a domain $\Omega$ of $\mathbb{R}^{3}$ and is surrounded by an insulating environment, e.g. air. In this case $u_{\varepsilon}$ represents the magnetic field $\left(u_{\varepsilon}=H_{\varepsilon}\right),-f$ is a prescribed electric current field supported outside $\Omega$, and $A$ is the electric resistance (that vanishes outside $\Omega$ ). $(8.1)_{1}$ then follows from the stationary Ampère and Faraday equations coupled with Ohm's laws:

$$
\nabla \times H_{\varepsilon}=J_{\varepsilon}, \quad \nabla \times E_{\varepsilon}=0, \quad E_{\varepsilon}=A \cdot\left(J_{\varepsilon}+f\right) \quad \text { a.e. in } \mathbb{R}^{3} .
$$

The resistance is here assumed to depend on the magnetic-field, as it is prescribed by the classical Hall effect, cf. e.g. [20]. The magnetic field $H_{\varepsilon}$ must also be related by a constitutive law to the magnetic induction $B_{\varepsilon}$, which is divergence free. If the magnetic permeability is scalar and uniform, i.e., $B_{\varepsilon}$ is proportional to $H_{\varepsilon}$, then we retrieve $(8.1)_{2}$.

Let $\Omega$ be a bounded Lipschitz domain of $\mathbb{R}^{3}$ and $A\left(=\left\{a_{i j}: i, j=1,2,3\right\}\right)$ a function $\mathbb{R}^{3} \times \Omega \times \mathcal{Y} \rightarrow \mathbb{R}^{9}$ such that

$A(v, \cdot, \cdot)$ is measurable w.r.t. the $\sigma$-algebra

generated by $\mathcal{B}(\Omega) \times \mathcal{L}(\mathcal{Y}), \quad \forall v \in \mathbb{R}^{3}$ 
(our discussion would be unchanged if $\mathcal{B}(\Omega) \times \mathcal{L}(\mathcal{Y})$ were replaced by $\mathcal{L}(\Omega) \times \mathcal{B}(\mathcal{Y})$ ), $v \mapsto A(v, x, y)$ is continuous, for any $x$ and a.a. $y$, $\exists c_{1}, c_{2} \in \mathbb{R}\left(0<c_{1}<c_{2}\right)$ such that for any $(v, x)$ and a.a. $y$ $c_{1} \xi_{i} \xi_{i} \leq a_{i j}(v, x, y) \xi_{i} \xi_{j}, \quad\left|\left\{a_{i j}(v, x, y) \xi_{i}\right\}\right| \leq c_{2}|\xi| \quad \forall \xi \in \mathbb{R}^{3}$.

The function $(v, x) \mapsto A\left(v, x, \frac{x}{\varepsilon}\right)$ is thus of Caratheodory class, and the mapping $x \mapsto A\left(v(x), x, \frac{x}{\varepsilon}\right)$ is measurable in $\Omega$ whenever so is the function $v$.

Let $f \in L^{2}(\Omega)^{3}$ and for any $\varepsilon>0$ consider the problem of finding $u_{\varepsilon} \in$ $H_{*}^{1}(\Omega)^{3}$ such that (8.1) is fulfilled in $\mathcal{D}^{\prime}(\Omega)$. By the compactness of the injection $H_{*}^{1}(\Omega)^{3} \rightarrow L^{2}(\Omega)^{3}$, it is easy to see that this equation has a solution.

Theorem 8.1. Assume that the conditions (8.2)-(8.4) are fulfilled. For any $\varepsilon>0$ let $u_{\varepsilon} \in H_{*}^{1}(\Omega)^{3}$ be a solution of $(8.1)$, and let $u_{1 \varepsilon}^{*} \in \ell^{2}\left(H_{*}^{1}(\mathcal{Y})^{3}\right)$ be such that (extending $u_{\varepsilon}$ with vanishing value outside $\Omega$ and omitting restrictions)

$$
\begin{aligned}
& \nabla \cdot u_{1 \varepsilon}^{*}(m, y)=0 \quad \text { for a. } a . y \in \mathcal{Y} \\
& \int_{\mathcal{Y}}\left[\nabla \times u_{1 \varepsilon}^{*}(m, y)-\left(\nabla \times u_{\varepsilon}\right)(\varepsilon(m+y))\right] \cdot \nabla \times \zeta d y=0 \quad \forall \zeta \in H^{1}(\mathcal{Y})^{3} .
\end{aligned}
$$

(By Lemma 4.1 this problem has a unique solution.) Let us set

$$
\begin{aligned}
u_{1 \varepsilon}(\varepsilon(m+y)) & :=u_{1 \varepsilon}^{*}(m, y) \\
z_{\varepsilon}(\varepsilon(m+y)) & :=\nabla \times u_{1 \varepsilon}^{*}(m, y) \quad \text { for a.a. } y \in Y, \forall m \in \mathbb{Z}^{3} .
\end{aligned}
$$

Then there exist $u \in H_{*}^{1}(\Omega)^{3}$ and $u_{1} \in L^{2}\left(\Omega ; H_{*}^{1}(\mathcal{Y})^{3}\right)$ such that, as $\varepsilon \rightarrow 0$ along a suitable subsequence,

$$
\begin{array}{rlrl}
u_{\varepsilon} & \rightarrow u & & \text { in } H_{*}^{1}(\Omega)^{3}, \\
u_{1 \varepsilon} \underset{2}{\rightarrow} u_{1}, z_{\varepsilon} \underset{2}{2} \nabla_{y} \times u_{1} & & \text { in } L^{2}(\Omega \times \mathcal{Y})^{3}, \\
\nabla \times u_{\varepsilon} \underset{2}{\rightarrow} \nabla \times u+\nabla_{y} \times u_{1} & & \text { in } L^{2}(\Omega \times \mathcal{Y})^{3} .
\end{array}
$$

This entails that

$$
\begin{array}{r}
\iint_{\Omega \times \mathcal{Y}}\left\{A(u(x), x, y) \cdot\left[\nabla \times u(x)+\nabla_{y} \times u_{1}(x, y)+f(x)\right]\right\} \\
{\left[\nabla \times v(x)+\nabla_{y} \times v_{1}(x, y)\right] d x d y=0} \\
\forall v \in H_{*}^{1}(\Omega)^{3}, \forall v_{1} \in L^{2}\left(\Omega ; H^{1}(\mathcal{Y})^{3}\right)
\end{array}
$$

By the arbitrariness of $v$ and $v_{1}$, the equation (8.10) is equivalent to the system

$$
\begin{aligned}
& \nabla \times \int_{\mathcal{Y}}\left\{A(u(x), x, y) \cdot\left[\nabla \times u(x)+\nabla_{y} \times u_{1}(x, y)+f(x)\right]\right\} d y=0 \text { in } \mathcal{D}^{\prime}(\Omega)^{3} \\
& \nabla_{y} \times\left\{A(u(x), x, y) \cdot\left[\nabla \times u(x)+\nabla_{y} \times u_{1}(x, y)+f(x)\right]\right\}=0 \text { in } \mathcal{D}^{\prime}(\Omega \times \mathcal{Y})^{3} .
\end{aligned}
$$


Proof. In this argument all fields defined in $\Omega$ are extended to $\mathbb{R}^{3}$ with vanishing value. Multiplying (8.1) by $u_{\varepsilon}$ it is easy to see that the family $\left\{u_{\varepsilon}\right\}$ is uniformly bounded in $H_{*}^{1}(\Omega)^{3}$. Theorem 3.2 then yields (8.7), (8.8) and

$$
\nabla \times u_{\varepsilon} \underset{2}{\rightarrow} \nabla \times u+\nabla_{y} \times u_{1} \quad \text { in } L^{2}(\Omega \times \mathcal{Y})^{3},
$$

as $\varepsilon$ vanishes along a suitable sequence. Thus $u_{\varepsilon} \rightarrow u$ in $L^{2}(\Omega)^{3}$, whence

$$
A\left(u_{\varepsilon}(x), x, \frac{x}{\varepsilon}\right) \underset{2}{\rightarrow} A(u(x), x, y) \quad \text { in } L^{2}(\Omega \times \mathcal{Y})^{9} .
$$

For any $v \in \mathcal{D}(\Omega)^{3}$ and any $v_{1} \in \mathcal{D}(\Omega \times \mathcal{Y})^{3}$, multiplying (8.1) by $v(x)+\varepsilon v_{1}\left(x, \frac{x}{\varepsilon}\right)$ and integrating in $\Omega$ we get

$$
\begin{aligned}
& \iint_{\Omega \times \mathcal{Y}}\left\{A\left(u_{\varepsilon}(x), x, \frac{x}{\varepsilon}\right) \cdot\left[\nabla \times u_{\varepsilon}(x)+f(x)\right]\right\} \\
& \cdot\left[\nabla \times v(x)+\varepsilon \nabla_{x} \times v_{1}\left(x, \frac{x}{\varepsilon}\right)+\nabla_{y} \times v_{1}\left(x, \frac{x}{\varepsilon}\right)\right] d x d y=0 .
\end{aligned}
$$

Passing to the limit in (8.1) we then get (8.10). The equations (8.11) are direct consequences of $(8.1)_{2}$ and $(8.5)_{1}$.

Let us now come to (8.9). By (8.7), denoting by $\varepsilon^{\prime}$ a suitable subsequence, $A\left(u_{\varepsilon^{\prime}}(x), x, \frac{x}{\varepsilon^{\prime}}\right) \underset{2}{\rightarrow} A(u(x), x, y)$ a.e. in $\Omega \times \mathcal{Y}$. Let us set $g_{\varepsilon^{\prime}}:=\nabla \times u_{\varepsilon^{\prime}}$ and $g:=\nabla \times u+\nabla_{y} \times u_{1}$. Recalling the upper estimate of (8.4), the two-scale Vitali theorem (cf. Lemma 2.3) then yields

$$
\int_{\Omega}\left\{\left[A\left(u_{\varepsilon^{\prime}}(x), x, \frac{x}{\varepsilon^{\prime}}\right) \cdot g_{\varepsilon^{\prime}}(x)\right] \cdot g_{\varepsilon^{\prime}}(x)-\left[A\left(u(x), x, \frac{x}{\varepsilon^{\prime}}\right) \cdot g_{\varepsilon^{\prime}}(x)\right] \cdot g_{\varepsilon^{\prime}}(x)\right\} d x \rightarrow 0 .
$$

By (8.10) and (8.14) (here with $v=g_{\varepsilon^{\prime}}$ and $v_{1} \equiv 0$ ) we then get

$$
\left.\int_{\Omega}\left[A\left(u(x), x, \frac{x}{\varepsilon^{\prime}}\right) \cdot g_{\varepsilon^{\prime}}(x)\right] \cdot g_{\varepsilon^{\prime}}(x) d x \rightarrow \iint_{\Omega \times \mathcal{Y}}[A(u(x), x, y) \cdot g(x, y)] \cdot g(x, y)\right\} d x d y .
$$

Notice that

$$
\begin{aligned}
v & \mapsto \int_{\Omega}\left[A\left(u(x), x, \frac{x}{\varepsilon^{\prime}}\right) \cdot v(x)\right] \cdot v(x) d x, \\
w & \mapsto \iint_{\Omega \times \mathcal{Y}}[A(u(x), x, y) \cdot w(x, y)] \cdot w(x, y) d x d y
\end{aligned}
$$

are the square of uniformly convex norms over $L^{2}(\Omega)^{3}$ and $L^{2}(\Omega \times \mathcal{Y})^{3}$, respectively. By a two-scale extension of the property of compactness by strict convexity [40], (8.12) then yields (8.9) for the subsequence $\left\{g_{\varepsilon^{\prime}}\right\}$. This actually holds for the whole sequence $\left\{g_{\varepsilon}\right\}$, since the same argument applies to any subsequence. 
Other quasilinear elliptic equations. If the magnetic induction $w_{\varepsilon}=B_{\varepsilon}$ is a nonlinear function of the magnetic field $u_{\varepsilon}$, then (8.1) must be coupled with the condition $\nabla \cdot w_{\varepsilon} \equiv 0$. If $A$ is independent of $u_{\varepsilon}$, for any $\varepsilon$ existence of a solution of the corresponding system can be proved via a procedure based on Murat's and Tartar's compensated compactness and on compactness by strict convexity. As $\varepsilon$ vanishes, convergence to a homogenized problem may then be derived via two-scale extensions of those compactness techniques, cf. [39], using the spaces $L_{\text {rot }}^{2}\left(\mathbb{R}^{3}\right)^{3}$ and $L_{\text {div }}^{2}\left(\mathbb{R}^{3}\right)^{3}$, and applying of the results of Sections 4,5 .

The analogous of the system (8.1) for the gradient operator reads

$$
-\nabla \cdot\left[A\left(u_{\varepsilon}(x), x, \frac{x}{\varepsilon}\right) \cdot\left(\nabla u_{\varepsilon}+f\right)\right]=0 \quad \text { in } \Omega ;
$$

here $u_{\varepsilon}$ is a scalar function, and no further condition like $(8.1)_{2}$ is needed. If $u_{\varepsilon}$ is interpreted as a temperature field, this may represent thermal equilibrium in a heterogeneous and anisotropic material, with a temperature-dependent heat conductivity tensor $A$. It is easy to see that this equation has at least one solution. This problem has been studied in a large literature, see the monographs quoted in the Introduction. The two-scale limit behaviour as $\varepsilon$ vanishes may be treated along the lines of the procedure that we used for (8.1).

Finally let us come to equations of the form

$$
\begin{aligned}
\nabla \times\left[A\left(\nabla \times u_{\varepsilon}(x), x, \frac{x}{\varepsilon}\right) \cdot \nabla \times u_{\varepsilon}\right]=f & \text { in } \Omega, \\
-\nabla \cdot\left[A\left(\nabla u_{\varepsilon}(x), x, \frac{x}{\varepsilon}\right) \cdot \nabla u_{\varepsilon}\right]=f & \text { in } \Omega .
\end{aligned}
$$

For each of them existence of a solution may be proved, provided that the corresponding operator is monotone; if it is even cyclically monotone, then this is the Euler equation of a convex functional, and variational techniques may be used, cf. [40]. More general quasi-linear equations will be studied apart.

\section{Conclusions}

Let a sequence $\left\{u_{\varepsilon}\right\}$ be such that $u_{\varepsilon} \overrightarrow{2} u$ in $L^{2}\left(\mathbb{R}^{N} \times \mathcal{Y}\right)^{M}$, and $P: \operatorname{Dom}(P) \rightarrow$ $L^{2}\left(\mathbb{R}^{N}\right)^{\tilde{M}}$ be a first-order differential operator (with constant coefficients), for suitable integers $M, \tilde{M}$. In each of the five cases that we dealt with in Sections 2-6, we constructed a sequence $\left\{u_{1 \varepsilon}\right\}$ such that, as $\varepsilon$ vanishes along a suitable subsequence, defining $\bar{u}$ as in (1.7),

$$
u_{1 \varepsilon} \underset{2}{\rightarrow} u_{1}, \quad \varepsilon P u_{1 \varepsilon} \underset{2}{\rightarrow} P_{y} u_{1}, \quad P u_{\varepsilon} \underset{2}{\rightarrow} P \bar{u}+P_{y} u_{1} \quad \text { in } L^{2}\left(\mathbb{R}^{N} \times \mathcal{Y}\right)^{\tilde{M}}
$$

here $\bar{u}$ is the weak one-scale limit of $\left\{u_{\varepsilon}\right\}, P_{y}$ is the version of $P$ acting on the variable $y$. For instance, in Section $2, M=1, \tilde{M}=N, P=\nabla, \operatorname{Dom}(P)=$ $H^{1}\left(\mathbb{R}^{N}\right)$; in Section $4, M=\tilde{M}=3, P=\nabla \times, \operatorname{Dom}(P)=L_{\text {rot }}^{2}\left(\mathbb{R}^{3}\right)^{3}$. 
We encountered two basic situations. In Sections 2, 3 the domain of $P$ has compact injection in $L^{2}\left(\mathbb{R}^{N}\right)^{M}$, so that $u_{\varepsilon} \rightarrow u$ in this space; the two-scale limit function then coincides with $u$, which does not depend on the fine-scale variable $y$. On the other hand in Sections 4, 5, 6 the sequence does not strongly one-scale converge in $L^{2}\left(\mathbb{R}^{N}\right)^{M}$, so that the two-scale limit function $u$ may depend on $y$; however of course $u_{\varepsilon} \rightarrow \bar{u}$ in $L^{2}\left(\mathbb{R}^{N}\right)^{M}$. We then represented the weak two-scale limit of $P u_{\varepsilon}$ in the form $P \bar{u}+P_{y} u_{1}$; this entails that the finescale information are confined to the term $\nabla_{y} \times u_{1}$. Notice that $P \bar{u}$ and $P_{y} u_{1}$ are orthogonal in $L^{2}\left(\mathbb{R}^{N} \times \mathcal{Y}\right)^{M}$.

An alternative representation of the form $P u+P_{y} \tilde{u}_{1}$ for the curl and divergence operators was studied by Wellander and Kristensson [41], who used a technique that is reminiscent of that of Theorem 3 of [27] and Proposition 1.14 of [1] for the gradient.

Remark added in proofs. Here we briefly compare the latter alternative representation of the two-scale limit of the curl with that of Section 4. By Theorem $4.2 \nabla_{y} \times u=0$, hence there exists $\varphi \in L^{2}\left(\mathbb{R}^{3} ; H_{*}^{1}(\mathcal{Y})\right)$ such that $u(x, y)=\bar{u}(x)+\nabla_{y} \varphi(x, y)$ for a.a. $(x, y) \in \mathbb{R}^{3} \times \mathcal{Y}$. Thus

$$
\nabla \times \bar{u}=\nabla_{x} \times u-\nabla_{x} \times \nabla_{y} \varphi=\nabla_{x} \times u+\nabla_{y} \times \nabla_{x} \varphi \quad \text { in } \mathcal{D}^{\prime}\left(\mathbb{R}^{3} \times \mathcal{Y}\right)^{3} .
$$

Setting $u_{1}^{*}:=u_{1}+\nabla_{x} \varphi \in H^{-1}\left(\mathbb{R}^{3} ; H_{*}^{1}(\mathcal{Y})^{3}\right)$ we then infer that

$$
\nabla \times \bar{u}+\nabla_{y} \times u_{1}=\nabla_{x} \times u+\nabla_{y} \times\left(\nabla_{x} \varphi+u_{1}\right)=\nabla_{x} \times u+\nabla_{y} \times u_{1}^{*} .
$$

However it is not obvious that $\nabla_{x} \times u \in L^{2}\left(\mathbb{R}^{3} \times \mathcal{Y}\right)^{3}$, and thus the latter equality a priori just holds in $H^{-1}\left(\mathbb{R}^{3} ; L^{2}(\mathcal{Y})^{3}\right)$; in other terms, both $\nabla_{x} \times u$ and $\nabla_{y} \times u_{1}^{*}$ might be elements of the latter space.

An analogous remark concerning the representation of the two-scale limit of the divergence applies to Theorems 5.2 and 6.1 .

Acknowledgements. This research was supported by the projects "Mathematical modelling and analysis of free boundary problems" and "Free boundary problems, phase transitions and models of hysteresis" of Italian M.I.U.R.. The author also gratefully acknowledges a useful talk with Stephan Luckhaus.

\section{References}

[1] Allaire, G., Homogenization and two-scale convergence. S.I.A.M. J. Math. Anal. 23 (1992), 1482 - 1518.

[2] Allaire, G., Shape Optimization by the Homogenization Method. New York: Springer 2002. 
[3] Allaire, G. and Conca, C., Bloch wave homogenization and spectral asymptotic analysis. J. Math. Pures Appl. 77 (1998), $153-208$.

[4] Arbogast, T., Douglas, J. and Hornung, U., Derivation of the double porosity model of single phase flow via homogenization theory. S.I.A.M. J. Math. Anal. 21 (1990), $823-836$.

[5] Bakhvalov, N. and Panasenko, G., Homogenization: Averaging Processes in Periodic Media. Dordrecht: Kluwer 1989.

[6] Bensoussan, G., Lions, J.-L. and Papanicolaou, G., Asymptotic Analysis for Periodic Structures. Amsterdam: North-Holland 1978.

[7] Bossavit, A., Griso, G. and Miara, B., Modelling of periodic electromagnetic structures. Bianisotropic materials with memory. J. Math. Pures. Appl. 84 (2005), $819-850$.

[8] Bourgeat, A., Luckhaus, S. and Mikelić, A., Convergence of the homogenization process for a double-porosity model of immiscible two-phase flow. S.I.A.M. J. Math. Anal. 27 (1996), 1520-1543

[9] Braides, A. and Defranceschi, A., Homogenization of Multiple Integrals. Oxford: Oxford University Press 1998.

[10] Casado-Díaz, J., Two-scale convergence for nonlinear Dirichlet problems in perforated domains. Proc. Royal Soc. Edinburgh A130 (2000), 249 - 276.

[11] Casado-Díaz, J. and Gayte, I.: A general compactness result and its application to two-scale convergence of almost periodic functions. C.R. Acad. Sci. Paris, Ser. I, 323 (1996), 329 - 334.

[12] Casado-Díaz, J., Luna-Laynez, M. and Martin, J. D., An adaptation of the multi-scale method for the analysis of very thin reticulated structures. C.R. Acad. Sci. Paris, Ser. I, 332 (2001), 223 - 228.

[13] Cioranescu, D., Damlamian, A. and Griso, G., Periodic unfolding and homogenization. C.R. Acad. Sci. Paris, Ser. I, 335 (2002), 99 - 104.

[14] Cioranescu, D. and Donato, P., An Introduction to Homogenization. New York: Oxford Univ. Press 1999.

[15] Conca, C., Planchard, J. and Vanninathan, M., Fluids and Periodic Structures. Paris: Wiley, Chichester and Masson 1995.

[16] Dal Maso, G., An Introduction to Г-Convergence. Boston: Birkhäuser 1993.

[17] Dautray, R. and Lions, J.-L., Analyse Mathématique et Calcul Numérique. Paris: Masson 1988.

[18] E, Weinan, Homogenization of linear and nonlinear transport equations. Comm. Pure Appl. Math. 45 (1992), 301 - 326.

[19] Jikov, V. V., Kozlov, S. M. and Oleŭnik, O. A., Homogenization of Differential Operators and Integral Functionals. Berlin: Springer 1994.

[20] Landau, L. and Lifshitz, E., Electrodynamics of Continuous Media. Oxford: Pergamon Press 1960. 
[21] Lenczner, M., Homogénéisation d'un circuit électrique. C.R. Acad. Sci. Paris, Ser. II, 324 (1997), $537-542$.

[22] Lenczner, M. and Senouci-Bereski, G., Homogenization of electrical networks including voltage-to-voltage amplifiers. Math. Models Meth. Appl. Sci. 9 (1999), $899-932$.

[23] Lions, J.-L., Some Methods in the Mathematical Analysis of Systems and their Control. Beijing: Science Press, and New York: Gordon and Breach 1981.

[24] Lions, J.-L. and Magenes, E., Non-Homogeneous Boundary Value Problems and Applications. Vol. I. Berlin: Springer 1972 (Paris: Dunod, 1968; French edition).

[25] Lukkassen, D., Nguetseng, G. and Wall, P., Two-scale convergence. Int. J. Pure Appl. Math. 2 (2002), $35-86$.

[26] Murat, F. and Tartar, L., H-convergence. In: Topics in the Mathematical Modelling of Composite Materials (eds.: A. Cherkaev, R. Kohn). Boston: Birkhäuser 1997, pp. $21-44$.

[27] Nguetseng, G., A general convergence result for a functional related to the theory of homogenization. S.I.A.M. J. Math. Anal. 20 (1989), $608-623$.

[28] Nguetseng, G., Asymptotic analysis for a stiff variational problem arising in mechanics. S.I.A.M. J. Math. Anal. 21 (1990), $1394-1414$.

[29] Sanchez-Palencia, E., Non-Homogeneous Media and Vibration Theory. New York: Springer 1980.

[30] Tartar, L., Course Peccot. Paris: Collège de France 1977 (unpublished, partially written in $[26])$.

[31] Tartar, L., Estimations des coefficients homogénéisés. In: Computing Methods in Applied Sciences and Engineering (eds.: R. Glowinski, J. L. Lions). Lecture Notes Math. 704. Springer 1979, pp. $364-373$.

[32] Tartar, L., Quelques remarques sur l'homogénéisation. In: Functional Analysis and Numerical Analysis (Proc. Japan-France Seminar 1976; ed.: H. Fujita). Japan Soc. Prom. Science 1978, pp. $468-482$.

[33] Tartar, T., Mathematical tools for studying oscillations and concentrations: from Young measures to H-measures and their variants. In: Multiscale Problems in Science and Technology. (eds.: N. Antonić et al.) Berlin: Springer 2002, pp. $1-84$.

[34] Visintin, A., Some properties of two-scale convergence. Rendic. Accad. Lincei XV (2004), $93-107$.

[35] Visintin, A., Towards a two-scale calculus. E.S.A.I.M. Control Optim. Calc. Var. 12 (2006), $371-397$.

[36] Visintin, A., Homogenization of doubly-nonlinear equations. Rendic. Accad. Lincei. 17 (2006), $211-222$.

[37] Visintin, A., Homogenization of the nonlinear Kelvin-Voigt model of viscoelasticity and of the Prager model of elasto-plasticity. Continuum Mech. Thermodyn. 18 (2006), $223-252$. 
[38] Visintin, A., Homogenization of the nonlinear Maxwell model of visco-elasticity and of the Prandtl-Reuss model of elasto-plasticity (submitted).

[39] Visintin, A., Electromagnetic processes in doubly-nonlinear composites (submitted).

[40] Visintin, A., Two-scale convergence of some integral functionals. Calc. Var. Partial Differential Equations (in press).

[41] Wellander, N. and Kristensson, G., Homogenization of the Maxwell equations at fixed frequency. S.I.A.M. J. Appl. Math. 64 (2003), $170-195$.

[42] Zhikov, V. V., On an extension of the method of two-scale convergence and its applications. Sb. Math. 191 (2000), $973-1014$.

Received December 20, 2004; revised June 30, 2006 